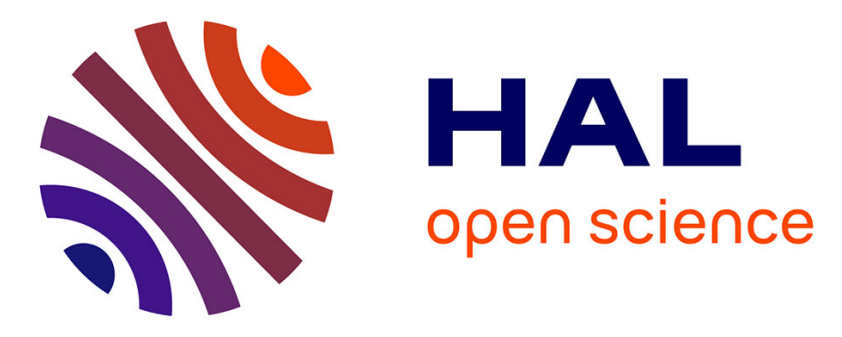

\title{
Are the Moon's Nearside-Farside Asymmetries the Result of a Giant Impact?
}

Meng-hua Zhu, Kai Wünnemann, Ross W.K. Potter, Thorsten Kleine, Alessandro Morbidelli

\section{- To cite this version:}

Meng-hua Zhu, Kai Wünnemann, Ross W.K. Potter, Thorsten Kleine, Alessandro Morbidelli. Are the Moon's Nearside-Farside Asymmetries the Result of a Giant Impact?. Journal of Geophysical Research. Planets, 2019, 124 (8), pp.2117-2140. 10.1029/2018JE005826 . hal-02396673

\section{HAL Id: hal-02396673 https://hal.science/hal-02396673}

Submitted on 5 Jan 2022

HAL is a multi-disciplinary open access archive for the deposit and dissemination of scientific research documents, whether they are published or not. The documents may come from teaching and research institutions in France or abroad, or from public or private research centers.
L'archive ouverte pluridisciplinaire HAL, est destinée au dépôt et à la diffusion de documents scientifiques de niveau recherche, publiés ou non, émanant des établissements d'enseignement et de recherche français ou étrangers, des laboratoires publics ou privés.

$$
\text { Copyright }
$$




\section{RESEARCH ARTICLE 10.1029/2018JE005826 \\ Key Points: \\ - Giant impact on the nearside of the Moon can reproduce the crustal thickness asymmetry between the nearside and farside \\ - The nearside lowlands and KREEP materials on the lunar surface can be explained by the giant impact scenario \\ - Giant impact predicts that the Moon has a significant ${ }^{182} \mathrm{~W}$ anomaly after the Moon formation, as expected by the collision formation of the Moon}

Supporting Information:

- Supporting Information S1

Correspondence to:

M.-H. Zhu,

mhzhu@must.edu.mo

Citation:

Zhu, M.-H., Wünnemann, K., Potter, R. W. K., Kleine, T., \& Morbidelli, A. (2019). Are the Moon's nearside-farside asymmetries the result of a giant impact?. Journal of Geophysical Research: Planets, 124, 2117-2140. https://doi.org/10.1029/2018JE005826

Received 11 SEP 2018

Accepted 19 APR 2019

Accepted article online 20 MAY 2019

Published online 12 AUG 2019

(C)2019. American Geophysical Union. All Rights Reserved.

\section{Are the Moon's Nearside-Farside Asymmetries the Result of a Giant Impact?}

\author{
Meng-Hua Zhu' ${ }^{1,2,3}$ (D, Kai Wünnemann ${ }^{2,7}$, Ross W.K. Potter ${ }^{4}$ iD, Thorsten Kleine ${ }^{5}$, \\ and Alessandro Morbidelli ${ }^{6}$ iD
}

${ }^{1}$ State Key Laboratory of Lunar and Planetary Sciences, Macau University of Science and Technology, Taipa, Macau, ${ }^{2}$ Museum für Naturkunde, Leibniz Institute for Evolution and Biodiversity Science, Berlin, Germany, ${ }^{3}$ CAS Center for Excellence in Comparative Planetology, Hefei, China, ${ }^{4}$ Department of Earth, Environmental and Planetary Sciences, Brown University, Providence, RI, USA, ${ }^{5}$ Institut für Planetologie, University of Münster, Münster, Germany, ${ }^{6}$ Département Lagrange, University of Nice-Sophia Antipolis, CNRS, Observatoire de la Côte d'Azur, Nice, France, ${ }^{7}$ Freie Universität Berlin, Institute of Geological Sciences, Planetary Sciences and Remote Sensing, Berlin, Germany

Abstract The Moon exhibits striking geological asymmetries in elevation, crustal thickness, and composition between its nearside and farside. Although several scenarios have been proposed to explain these asymmetries, their origin remains debated. Recent remote sensing observations suggest that (1) the crust on the farside highlands consists of two layers: a primary anorthositic layer with thickness of $\sim 30-50$ $\mathrm{km}$ and on top a more mafic-rich layer $\sim 10 \mathrm{~km}$ thick and (2) the nearside exhibits a large area of low-Ca pyroxene that has been interpreted to have an impact origin. These observations support the idea that the lunar nearside-farside asymmetries may be the result of a giant impact. Here using quantitative numerical modeling, we test the hypothesis that a giant impact on the early Moon can explain the striking differences in elevation, crustal thickness, and composition between the nearside and farside of the Moon. We find that a large impactor, impacting the current nearside with a low velocity, can form a mega-basin and reproduce the characteristics of the crustal asymmetry and structures comparable to those observed on the current Moon, including the nearside lowlands and the farside's mafic-rich layer on top of a primordial anorthositic crust. Our model shows that the excavated deep-seated KREEP (potassium, rare earth elements, and phosphorus) material, deposited close to the basin rim, slumps back into the basin and covers the entire basin floor; subsequent large impacts can transport the shallow KREEP material to the surface, resulting in its observed distribution. In addition, our model suggests that prior to the asymmetry-forming impact, the Moon may have had an ${ }^{182} \mathrm{~W}$ anomaly compared to the immediate post-giant impact Earth's mantle, as predicted if the Moon was created through a giant collision with the proto-Earth.

Plain Language Summary Beginning with the Apollo era, spacecraft observations have shown that the Moon has striking asymmetries between its nearside and farside in topography, crustal thickness, and composition. These asymmetries are likely a product of very early geological processes on the Moon. Understanding their formation mechanism may help to constrain models of global lunar evolution and magma-ocean crystallization. Several hypotheses have been suggested, though none can explain the observations satisfactorily.

Recent spacecraft observations from the Gravity Recovery and Interior Laboratory (GRAIL) mission indicate that the farside crust is $\sim 20$-km thicker than the nearside crust, and remote sensing data show that this extra crust is composed of a mafic-rich layer covering the primary crust. This layered crustal structure on the farside, together with a large area of low-Ca pyroxene on the nearside observed by Kaguya mission that was explained to be formed via impact through melting a mixture of crust and mantle materials, means that a giant impact on the nearside may explain the nearside-farside asymmetries. To investigate this possibility, we quantitatively studied the giant impact theory using numerical modeling. Our models confirm that a giant impact on the nearside can explain nearside-farside asymmetries.

We demonstrate that a large body slowly impacting the nearside of the Moon can reproduce the observed crustal thickness asymmetry and form both the farside highlands and the nearside lowlands. Additionally, the model shows that the resulting impact ejecta would cover the primordial anorthositic crust to form a two-layer crust on the farside, as observed. Overall, the modeling results are generally in agreement with assumptions that are based observations and provide credible explanations for the observed asymmetries in crustal thickness and elevation. This work also provides a plausible explanation for the existence of KREEP 
(potassium, rare earth element, and phosphorus) on the lunar surface. A very important implication of this work is that it can explain the conundrum about isotopic differences between the Earth and Moon, particularly the significant anomaly of ${ }^{182} \mathrm{~W}$ in the Moon, as this anomaly would occur if this giant impact added material to the Moon after the initial Moon-forming. Our model can thus explain this isotope anomaly in the context of the giant impact scenario of the Moon's origin.

In summary, this work quantitatively supports the long-standing hypothesis that a giant impact resulted in the Moon's nearside-farside asymmetries and the Procellarum KREEP terrain was formed as a consequence of such an impact event. In addition, this work also provides a reference for reconstructing the early history of planetary bodies with similar asymmetries, such as Mars.

\section{Introduction}

Since the Apollo era, it has been known that the Moon has striking nearside-farside asymmetries in topography, crustal thickness, and chemical composition (Lawrence et al., 1998; Toksöz et al., 1974; Zuber et al., 1994). On the nearside, the Moon has a low topography and thin crust, whereas on the farside, the Moon has a high topography and thick crust. In contrast to the topographic and crustal thickness distribution between the nearside and farside, the Moon has a high concentration of the KREEP material on the nearside but shows a depletion of the KREEP material on the farside highlands (see Figure 1). As these asymmetries were likely established soon after the Moon formed, understanding their origin is key for shaping models of the Moon's early evolutionary history (Shearer, 2006), including the impact cratering process (Miljkovic et al., 2013; Potter et al., 2015; Zhu et al., 2017), volcanic activities (Hess \& Parmentier, 2001), and thermal evolution (Ghods \& Arkani-Hamed, 2007; Grimm, 2013; Laneuville et al., 2013; Parmentier et al., 2002; Wieczorek \& Phillips, 2000; Zhang et al., 2013; Zhong et al., 2000).

Several scenarios have been proposed to explain the possible origins of the nearside-farside asymmetries, including those invoked by external processes such as asymmetric nearside/farside cratering (Wood, 1973), ejecta deposition from South Pole-Aitken (SPA) basin (Zuber et al., 1994), early inhomogeneous tidal heating (Garrick-Bethell et al., 2010), and accretion of a companion moon (Jutzi \& Asphaug, 2011). Alternatively, thermochemical processes have been suggested as the origin of the nearside-farside asymmetries, such as the magma-ocean convective asymmetries (Loper \& Werner, 2002), asymmetric mantle contribution (Wasson \& Warren, 1980), and asymmetric crustal growth (Ohtake et al., 2012). In addition, the asymmetric distribution of crustal thickness is coupled with the occurrence of mare basalts over the lunar surface. The majority of mare basalts erupted on the nearside, whereas the mare basaltic eruptions on the farside are scarce (Lucey et al., 1998). Two possible scenarios, the degree-1 upwelling (Zhong et al., 2000) and downwelling (Parmentier et al., 2002) of ilmenite-rich cumulates, have been proposed to explain the nearside-farside asymmetric distribution of mare basalts over the lunar surface. However, as the formation time, bulk composition, crustal structures, and mantle evolution process of the Moon are not completely understood, the origin of the nearside-farside asymmetries remains enigmatic.

Recent observations from the Gravity Recovery and Interior Laboratory (GRAIL) mission suggest a crustal thickness of $\sim 30-40 \mathrm{~km}$ on the nearside and $\sim 50-60 \mathrm{~km}$ on the farside of the Moon (Wieczorek et al., 2013). The analysis of global distribution of major minerals on the lunar surface suggests that the thicker crust on the farside highlands consists of two layers: a primordial anorthositic crust $\sim 30-50 \mathrm{~km}$ thick with a more mafic-rich layer $\sim 10 \mathrm{~km}$ thick on top (Donaldson Hanna et al., 2014; Yamamoto et al., 2012). Simultaneously, a large area of the low-Ca pyroxene was observed on the nearside by the Kaguya spectral instrument (Nakamura et al., 2012). As the low-Ca pyroxene can be formed during an impact by melting a mixture of crust and mantle materials (Hess, 1994; Warren et al., 1996), the large area of low-Ca pyroxene on the nearside of the Moon was proposed to have an impact origin (Nakamura et al., 2012). According to the most accepted lunar magma ocean (LMO) model (e.g., Warren, 1985), the plagioclase feldspar floated to the top of magma ocean that produced the ferroan anorthosite crust and the substantially dense minerals cumulated at the bottom that formed the mafic mantle during the crystallization of magma ocean. The layered crustal structure (e.g., a more mafic-rich layer on top of the primordial anorthositic crust) on the farside (Donaldson Hanna et al., 2014; Yamamoto et al., 2012), together with the observation of a large area of impact-induced minerals (e.g., low-Ca pyroxene) on the nearside (Nakamura et al., 2012), may indicate that a giant impact on the nearside can explain the observed asymmetries. 


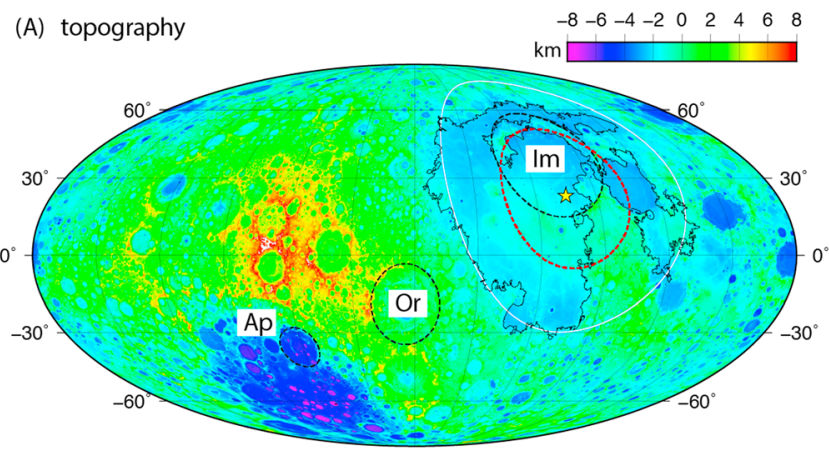

(B) crustal thickness

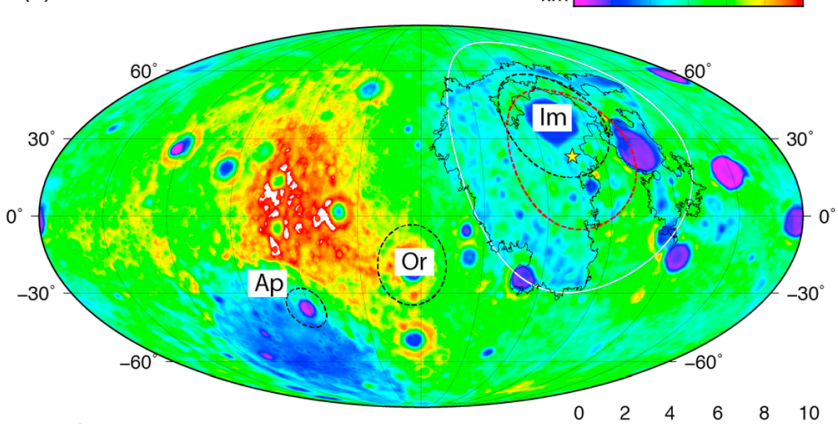

(C) thorium
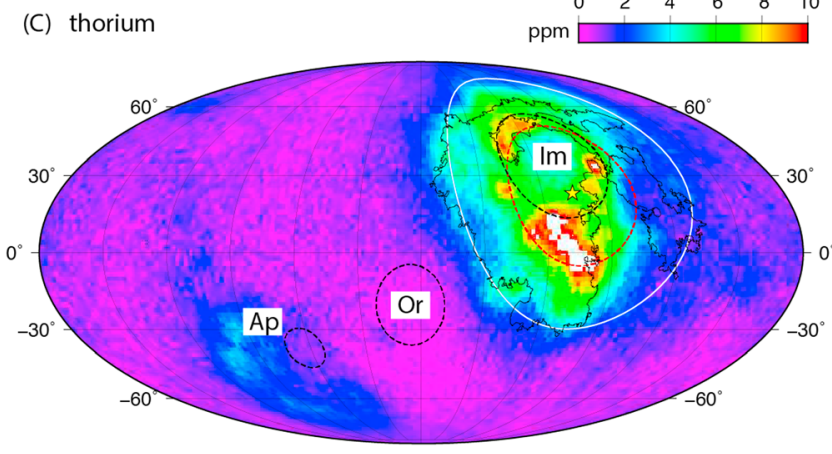

Figure 1. The (a) topographic, (b) crustal thickness, and thorium distribution of the Moon show a dichotomy between the nearside and farside. Data are presented in Mollweide equal-area projection centered at $90^{\circ} \mathrm{W}$ longitude, with nearside on the right of center and farside on the left of center. The star on the nearside represents the center of the proposed Procellarum basin (Wilhelms, 1987); the red dashed line and white solid line represent the boundary of transient crater with a radius of $850 \mathrm{~km}$ and final basin with a radius of 1,600 $\mathrm{km}$ of the proposed Procellarum basin, respectively (Wilhelms, 1987). The thin black line on the nearside of the Moon represents the Procellarum KREEP Terrane boundary. The black dashed lines represent the boundary of Imbrium (Im), Orientale (Or), and Apollo (Ap) basin, respectively. The topographic and crustal thickness distributiona are derived from the Lunar Orbiter Laser Altimeter (Smith et al., 2010) and Gravity Recovery and Interior Laboratory (GRAIL) observations (Wieczorek et al., 2013), respectively; the thorium distribution is from the observations of Lunar Prospector gamma ray spectrometer (Prettyman et al., 2006).
A giant impact on the nearside, creating a 3,200-km diameter Procellarum basin (e.g., Wilhelms \& McCauley, 1971; Wilhelms, 1987; Whitaker, 1981; see Figure 1) or a mega basin covering the whole nearside hemisphere (e.g., Byrne, 2007), has been proposed more than a decade, but until now this event has not been quantitatively assessed because no obvious structures for this mega basin are observed. The GRAIL data show Bouguer gravity anomalies (Zuber et al., 2013) at the central region of the nearside that have been interpreted as a gigantic tectonomagmatic structure (Andrews-Hanna et al., 2014), rather than a mega basin structure resulting from a giant impact. However, the mega basin structure may not be the same to the surface expression of a typical basin formed later. It is likely that the morphologies of mega basin were heavily modified and possibly even erased due to high internal temperature of the early Moon (Miljkovic et al., 2017), which easily allowed for isostatic relaxation processes (Freed et al., 2014). Therefore, it is highly possible that remnants of a giant impact on the early Moon may be less pronounced and significantly different from the impact basins formed later. To test whether a giant impact on the nearside is a plausible mechanism for the formation of Moon's asymmetries, we performed a systematic numerical modeling study and quantified the outcome of such impact events.

First, we investigate the collision probability of a Ceres-sized impactor (the estimated size range of the impactor in this study) on the early Moon (section 2). Then we introduce the giant impact simulation (section 3 ) and present how we determine the postimpact ejecta and crustal thickness after impact (section 4). In the results section (section 5) we present the impact cratering process, the basin size, and the crustal thickness distribution as a result of the giant impact event to see whether such a collision scenario could reproduce the global crustal thickness distribution of the Moon and the layered crustal structures on the farside highlands, as observed; next, we discuss whether such a giant impact event could form the lowlands on the nearside and explain the KREEP distribution on the Moon. Subsequently, we use our giant impact model as an attempt to explain the observed ${ }^{182} \mathrm{~W}$ anomaly in the Moon that is difficult to explain by the Moon-forming impact model (section 6). Plans for the future studies to further improve the proposed giant impact model for the formation of the nearside-farside asymmetry of the Moon are discussed in section 7 . Finally, we summarize our studies and provide conclusions (section 8).

\section{Collision Probability of a Ceres-Sized Impactor on the Moon}

The chemical analyses of derivative lunar mantle melts and crustal samples suggest that the Moon is extremely depleted in highly siderophile elements (HSEs; Ryder, 2002; Walker et al., 2004; Walker, 2009; Day et al., 2007 ; Day et al., 2010). The bulk HSE of the Moon, mainly attributed by the postcore-formation late accretion, corresponds to a mass of $\sim 2.1 \times 10^{19} \mathrm{~kg}$ for the chondritic material delivered to the Moon's mantle (Day et al., 2007; Day et al., 2010). For the late accretion, $\sim 60 \%$ of the impactor material is thought to be implanted in the Moon and the rest is vaporized and lost into space (Artemieva \& Shuvalov, 2008). Thus, the usual interpretation for the amount of HSEs is that the Moon accreted $\sim 3.5 \times 10^{19} \mathrm{~kg}$ of chondritic impactor material after its formation (Morbidelli et al., 2012). This amount of material corresponds to $\sim 1.5 \%$ of the current mass of the asteroid belt (Krasinsky et al., 2002). Assuming that the size frequency distribution of projectiles that hit the Moon was analog to that of main belt asteroids (Strom et al., 2005), the number of projectiles of any size that hit our 
satellite is $\sim 1.5 \%$ of the number of corresponding objects existing today in the belt. For a projectile with diameter of $\sim 800-900 \mathrm{~km}$ (Ceres-sized impactor), there is only one asteroid in the current main belt asteroid. Therefore, the probability of a Ceres-sized impactor hitting the Moon is $\sim 1.5 \%$. This probability is consistent with that estimated in Bottke et al. (2010).

However, the extrapolation backward in time of the decay of lunar bombardment rate over the last 4.1 Gyr (Neukum \& Ivanov, 1994) suggests that the Moon accreted a mass about 10 to 30 times larger than that inferred from the HSE content (Morbidell et al., 2018; Morbidelli et al., 2012). These two pieces of information can be reconciled if the lunar HSEs have been sequestered into the lunar core at the end of the crystallization of the LMO and mantle overturn, as proposed in Morbidell et al. (2018; see also Rubie et al., 2016). In this case, the HSE budget in the Moon would trace only the amount of chondritic material accreted after the LMO crystallization and not the material accreted since the formation of the Moon itself. If the LMO crystallization occurred late, as argued in Elkins-Tanton et al. (2011), the difference is relevant. The accretion of $3.5-10 \times 10^{20} \mathrm{~kg}$ of material (i.e., $\sim 10-30$ times larger than usually considered) can be reconciled with the lunar HSE budget if the LMO crystallized about 4.35 Ga ago (Morbidell et al., 2018). Obviously, if the total mass of projectiles hitting the Moon was 10-30 times larger than usually considered, the collision probability of a Ceres-sized impactor grows to $\sim 15 \%-45 \%$. Moreover, it is possible that the size frequency distribution of projectiles was a little different to that of main belt asteroids (Strom et al., 2005), in particular for large projectiles (Bottke et al., 2010, 2005; Marchi et al., 2014, 2018). A slight difference of the size frequency distribution can increase the collision probability of a Ceres-sized impactor to 1 or much bigger.

There is no reason, though, that the size distribution of the projectile that hit the Moon just after its formation was identical to that of main belt asteroids. The lunar projectiles were, in fact, in majority planetesimals left over from the terrestrial planet creation process, namely, a distinct reservoir from asteroids, so some differences in size distributions, particular at large sizes, can be expected (Morbidell et al., 2018; Morbidelli et al., 2012). A different calibration can be obtained by looking at Mars. The formation of the Borealis basin requires a projectile of about 1,500 km in diameter (Marinova et al., 2008). From the leftover of planet accretion, the Moon receives $\sim 1 / 2$ of the impacts of Mars (the ratio of collision probabilities between Mars and the Moon is 2; see Morbidell et al., 2018). Thus, the impact of an 800- to 900-km-sized body with the Moon is not unlikely. However, we cannot exclude that the Borealis impact occurred before the Moon-forming event and that no projectiles of comparable sizes were available after the Moon was formed.

\section{Simulation of Giant Impact Event}

We simulate the collision of large bodies with the early Moon using the iSALE shock-physics code (Dellen version; Collins et al., 2004; Wünnemann et al., 2006). We approximate the Moon by a 3,500-km-diameter sphere with a 40-km-thick crust (Wieczorek et al., 2013) on top of a 1,360-km-thick mantle and a 700-kmdiameter iron core at the center. We use the Tillotson equation of state for gabbroic anorthosite (Ahrens \& O'Keefe, 1977), the analytic equation of state for dunite (Benz et al., 1989), and iron (Thompson \& Lauson, 1972) to calculate the thermodynamic behavior of crustal, mantle, and core material, respectively. The initial densities of these materials are assumed to be 2.9 (crust), 3.3 (mantle), and $7.8 \mathrm{~g} / \mathrm{cm}^{3}$ (core). Due to the given size of the impactor with diameter larger than $300 \mathrm{~km}$, we assume that a differentiated structure (Lee \& Halliday, 1997) has a dunite mantle with density of $\sim 3.0 \mathrm{~g} / \mathrm{cm}^{3}(\sim 70 \%$ by mass) and an iron core with density of $7.8 \mathrm{~g} / \mathrm{cm}^{3}$ ( $\sim 30 \%$ by mass), similar to the average silicate-to-metal ratio for the terrestrial planets. We account for the material strength and dynamic fracturing by a constitutive model according to Collins et al. (2004) and Ivanov et al. (2010) in our simulations. We neglect the porosity inside the Moon, first, because that the porosity profiles (Besserer et al., 2014) derived from the current Moon may not represent the characteristics of the early Moon, and second a porous top layer (crust) of the Moon would not affect the basin formation process for a giant impact (Milbury et al., 2015). The material parameters used in the modeling are listed in Table 1.

Since the target's temperature gradient has a significant effect on the formation of large impact basins on the Moon (Ivanov et al., 2010; Miljkovic et al., 2013; Potter et al., 2015), we use two possible thermal gradients for the early Moon in our simulations (see Figure 2). Both temperature profiles consist of a near-surface conductive heat transfer portion (Potter et al., 2012) and a convective portion at depth (Spohn et al., 2001). Thermal 
Table 1

Material Parameters for iSALE-2-D Numerical Model Used in This Work

\begin{tabular}{|c|c|c|c|}
\hline Description & Crust (target) & Mantle (target/impactor) & Core (target/impactor) \\
\hline Equation of state & Gabbroic anorthosite Tillotson & Dunite ANEOS & Iron ANEOS \\
\hline Melt temperature at zero pressure $(\mathrm{K}), T_{m, 0}$ & 1,360 & 1,436 & 1,811 \\
\hline Constant in thermal softening law, $\varepsilon$ & 0.7 & 2.0 & 2.0 \\
\hline Constant in Simon approximation (GPa), $\alpha$ & 4.5 & 1.4 & 107 \\
\hline Exponent in Simon approximation, $c$ & 3.0 & 5.0 & 1.76 \\
\hline Poisson's ratio & 0.25 & 0.25 & 0.30 \\
\hline Cohesion (damaged; MPa) & 0.01 & 0.01 & \\
\hline Coefficient of internal friction for material (damaged) & 0.6 & 0.6 & \\
\hline Limiting strength at high pressure (damaged; GPa) & 2.5 & 3.5 & \\
\hline Cohesion (intact; MPa) & 20 & 50 & 100 \\
\hline Coefficient of internal friction for material (intact) & 1.4 & 1.5 & \\
\hline Limiting strength at high pressure (intact; GPa) & 2.5 & 3.5 & \\
\hline Minimum failure strain for low pressure states & $10^{-4}$ & $10^{-4}$ & \\
\hline Increase in failure strain with pressure & $10^{-11}$ & $10^{-11}$ & \\
\hline Pressure above which failure is always compressional (MPa) & 0.3 & 0.3 & \\
\hline$\gamma_{\eta}$ constant & 0.004 & 0.004 & \\
\hline$\gamma_{\beta}$ constant & 230 & 230 & \\
\hline Peak vibrational velocity as a fraction of the peak particle velocity & 0.1 & 0.1 & \\
\hline
\end{tabular}

profile 1 (TP1) has a crustal gradient of $30 \mathrm{~K} / \mathrm{km}$, and the mantle temperature follows an adiabatic gradient $(0.5 \mathrm{~K} / \mathrm{km})$ at temperatures in excess of 1,300 K (Freed et al., 2014). Thermal profile 2 (TP2) has a crust and upper mantle temperature gradient of $50 \mathrm{~K} / \mathrm{km}$; the temperature follows the mantle solidus between a depth of 40-350 km, which would cause partial melting of the upper mantle at this depth range; in the deep mantle ( $>350-\mathrm{km}$ depth) the temperature reaches $1,670 \mathrm{~K}$ and remains constant (Potter et al., 2012). In contrast with TP1, which represents the thermal gradient of a relatively cold Moon (Spohn et al., 2001), TP2 represents a

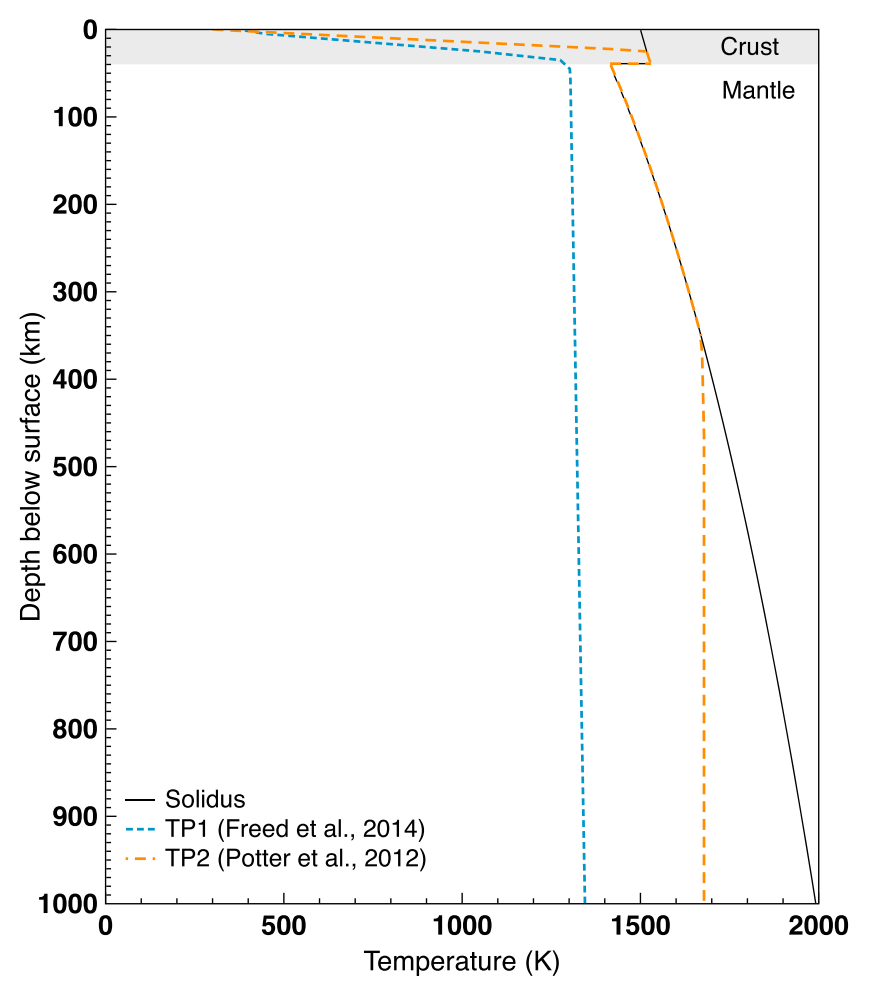

Figure 2. The thermal profiles of the Moon used in this work. Thermal profile 1 (TP1) represents the cold temperature profile of the Moon; TP2 represents the warm temperature profile. much warmer thermal profile of the Moon at the time of or before the SPA basin formation (Laneuville et al., 2013; Potter et al., 2012). For the proposed impact scenario, we consider TP2 as the more likely thermal state of the early Moon; however, to have some controls on the effect of the preimpact temperature on the basin formation, we also consider the rather cold TP1 model that may represent the present Moon. As the giant impact was thought to occur prior to the SPA-forming impact, it is possible that the LMO had not yet completely crystallized at the time of the giant impact, in which a thin melt-layer might be located between the mantle and the crust. However, as we know very little on the composition and thickness of this melt layer when the giant impact occurred, the temperature variation with depth in this layer is not well constrained. In addition, for the giant impact, the basin forming process and ejecta thickness distribution are not significantly sensitive to the temperature anomaly within a thin layer beneath the lunar crust (Zhu et al., 2017). Therefore, to be conservative, we assume that the temperature of the lower crust and upper mantle, as shown in TP2 profile, follows the solidus to a depth of $350 \mathrm{~km}$. The assumed thermal profiles are comparable to those used in other studies on the formation of lunar basins (e.g., Potter et al., 2013; Zhu et al., 2015); however, other temperature profiles that slightly differ at shallow depth (e.g., Miljkovic et al., 2013) are also possible.

The computational domain covers an area of 5,000 $\mathrm{km}$ in the lateral and $9,500 \mathrm{~km}$ in vertical direction in all models with a cell size of $10 \mathrm{~km}$ in the high-resolution zone $(500 \times 950$ cells $)$. In order to check whether the resolution is sufficient we also carried out some additional runs with a cell size of $5 \mathrm{~km}$ in the high-resolution zone. As the results of both resolutions (5-km cell size and 10-km cell size) are very similar on the basin 
size and ejecta thickness distribution, we use the lower resolution for the majority of models presented here, as the higher resolution models are computationally very expensive.

We set up all the models on a two-dimensional cylindrically symmetric grid, which allows for simulating the vertical impacts only. Two-dimensional models are preferred over three-dimensional models as the latter require far greater computational demands and do not allow for systematic high-resolution parameter studies. In addition, the vertical impacts provide a suitable proxy for impacts over a wide range of impact angles (e.g., Ivanov et al., 2010). As a consequence, the simulation of vertical impact is frequently used for the studies of the large basin formation on the Moon (e.g., Ivanov et al., 2010; Melosh et al., 2013; Miljkovic et al., 2013; Potter et al., 2012, 2013). However, it has been shown that craters tend to become elliptical at large angles with increasing crater efficiency (e.g., Collins et al., 2011; Elbeshausen et al., 2013). For the given size of the impactor, the crater efficiency is relatively low and we cannot rule out that an oblique impact may result in an elliptical crater and an asymmetric crustal thickness. Nevertheless, the 2-D vertical impact assumption serves as a first-order estimate to test the feasibility of an impact scenario. After significantly narrowed the range of possible impact parameters, the specific impact scenarios under the consideration of the impact angle will be studied in detail in the future (see the discussions in section 7).

We assume in our models that the gravity at the surface of the sphere representing the Moon corresponds to the average lunar gravitational acceleration of $1.62 \mathrm{~m} / \mathrm{s}^{2}$. The center of gravity is fixed and located at the center of the lunar sphere. We neglect that this initial gravity field may change slightly due to the redistribution of mass during crater formation. A self-consistent gravity model certainly is more realistic for the modeling of giant impact; however, such simulations are computationally very expensive and do not allow for a systematic parameter study where several hundred models are required. We find that a fixed gravity field causes some unrealistic oscillations of the target around the gravity center but consider the effect of such oscillations on the basin formation process to be small. Small oscillations in the gravity field do also not change the ejecta trajectories significantly (e.g., Ivanov et al., 2010; Potter et al., 2013). In addition, the similarities on the early stages of basin formation and ejecta emplacement for simulations with self-gravity and central gravity (see Figure S1 in the supporting information) suggest no significant differences on the ejecta trajectories as well. In conclusion we consider the effect of gravity variations as a consequence of crater formation to be small and, for the computational convenience, neglect this effect on the processes we investigate here for the given impact size range. However, with increasing impactor size changes in the gravity field related to the redistribution of mass become gradually more important. Further studies are required to better constrain the size ratio between the impactor and the target where a self-consistent gravity field has to be taken into account (see the discussions in section 7).

For the selected parameters, where the biggest uncertainties exist (e.g., the size and velocity of the impactor), we run models covering the entire plausible range: the impactor diameter is varied between 400 and 1,000 $\mathrm{km}$. The impact velocity is varied between 3 and $17 \mathrm{~km} / \mathrm{s}$ to cover a suitable range of impact velocities for the early Moon (Marchi et al., 2012). In total, we run 240 simulations and each run requires $\sim 1$ month of computation time. We also run models (120 simulations) with primitive impactors (no iron core), which do not differ too much from models where we include a metal core inside the impactor.

\section{Ejecta and Crustal Thickness Calculation of Giant Impact Event}

As we use the distribution of the ejecta on the surface of the Moon and related changes of the crustal thickness as important constraint of our models in comparison to observations (e.g., crustal thickness model derived from GRAIL gravity data, Wieczorek et al., 2013), we describe here how we derive these parameters, which are not directly provided by our models. We track the ejecta trajectories and their locations using Lagrangian tracers in iSALE. These tracers are initially placed in each computational cell and represent the matter originally in that cell throughout the simulation. When a tracer moves above the preimpact target surface, it is considered as ejecta and its launch time $\left(\boldsymbol{t}_{\mathrm{o}}\right)$, launch position $\left(\boldsymbol{p}_{\mathrm{o}}\right)$, and launch velocity $(\boldsymbol{V})$ are recorded (see Figure 3). We estimate the ejection angle as the angle to the local horizon. For example, for ejecta $\boldsymbol{a}$ with a launch velocity of $\boldsymbol{V}$, we calculate its normal velocity $\left(\boldsymbol{V}_{\boldsymbol{r}}\right)$ and tangent velocity $\left(\boldsymbol{V}_{\boldsymbol{t}}\right)$, from which the launch angle is estimated to be $\theta=\operatorname{atan}\left(\boldsymbol{V}_{\boldsymbol{r}} / \boldsymbol{V}_{\boldsymbol{t}}\right.$; see Figure 3a). This method has been used previously in several modeling studies on the ejecta distribution (e.g., Luther et al., 2018; Wünnemann et al., 2016). Although the ejecta behave more as a continuous flow with partly fluidized melt, they move along 

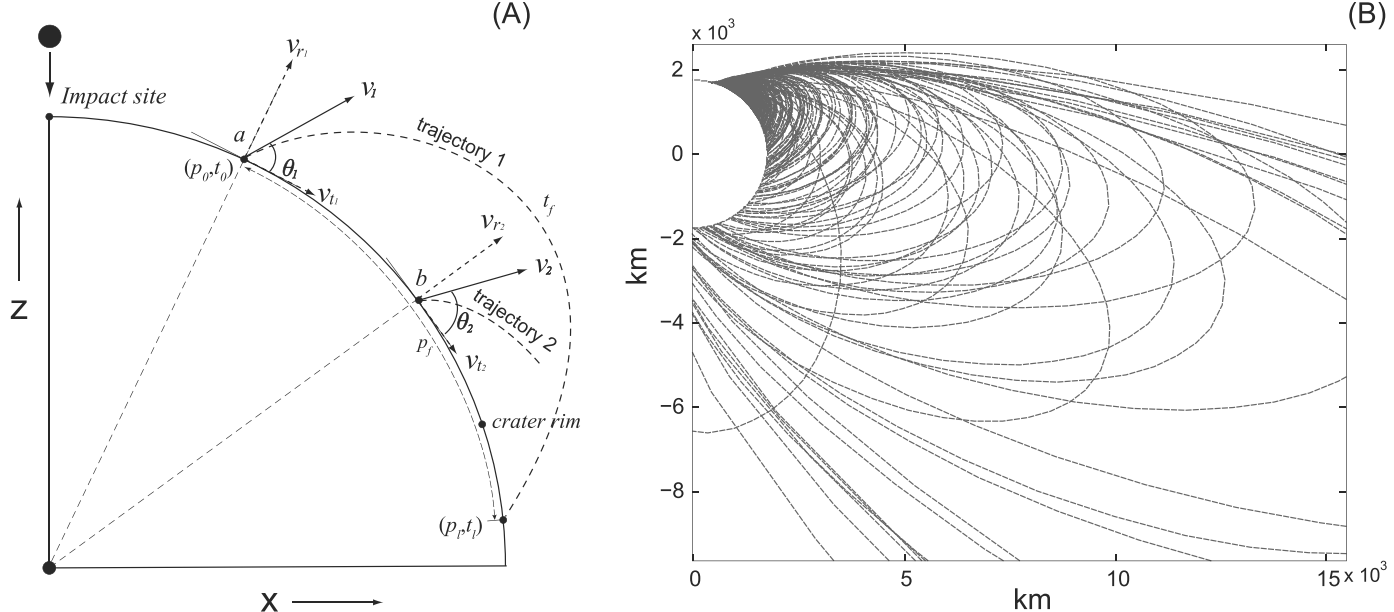

Figure 3. (a) The launch position $\left(p_{0}\right)$, velocity $(v)$, and angle $(\theta)$ estimation for the ejecta. The launch time $\left(t_{0}\right)$, landing time $\left(t_{l}\right)$, flight distance $\left(t_{f}\right)$, and landing position of the ejecta are also calculated. Note that $p_{f}$ defines the flight distance along the lunar surface. (b) The elliptical trajectory for each ejecta for the best fit model in this work.

parabolic trajectories and land on the lunar surface with approximately the same velocity as launched. Therefore, it is reasonable to use the hyperparabolic function to estimate their elliptical trajectory for the given ejecta angles and velocities (see Figure $3 \mathrm{~b}$; the elliptical ejecta trajectories for the best fit model in this work). The flight time $\left(\boldsymbol{t}_{f}\right)$, landing position $\left(\boldsymbol{p}_{l}\right)$, and landing time $\left(\boldsymbol{t}_{\boldsymbol{l}}=\boldsymbol{t}_{\boldsymbol{o}}+\boldsymbol{t}_{\boldsymbol{f}}\right)$ on the lunar surface are also calculated (Dobrovolskis, 1981; Hood \& Artemieva, 2008).

The method to calculate ejecta trajectories separately from the crater formation process by tracers allows to determine the landing site accurately but not the final deposition. However, it is not trivial to estimate the final location of ejecta because of two reasons. First of all, ejecta do not drop vertically on the ground but strike the surface with the angle and velocity that corresponds to the ejection angle and velocity. Therefore, ejecta may slide along the surface and mix with the local material. The process of entrainment of local material into ejecta blanket upon landing (e.g., Hörz et al., 1983) affects the ejecta stratigraphy significantly and is considered to be important for ejecta deposits several tens to hundred meters thick. For ejecta deposits resulting from large basin forming impacts several kilometers thick we consider this effect to be small and do not take into account here. Second, after the deposition of ejecta, ground motion as a result of the collapse of the transient cavity and subsequent modification process, such as inward slumping of large blocks, takes place and can change the final location of ejecta. The significance of postemplacement motion depends on the distance to the transient crater rim and is more important close to the rim of the transient crater where modification processes are much more pronounced than at some distance to the crater (Oberbeck, 1975). In order to take this effect into account, we first determine the flight distance of each tracer along the lunar surface to obtain the ejecta landing site. We then assume that each ejecta moves with the local (target) material around its landing site. For example, for ejecta with a launch position of $\boldsymbol{p}_{\boldsymbol{o}}$, launch time of $\boldsymbol{t}_{\boldsymbol{o}}$, and flight time of $\boldsymbol{t}_{\boldsymbol{f}}$, we estimate its landing position of $\boldsymbol{p}_{\boldsymbol{l}}=\boldsymbol{p}_{\boldsymbol{o}}$ $+\boldsymbol{p}_{\boldsymbol{f}}$; here $\boldsymbol{p}_{\boldsymbol{f}}$ is the flight distance along the lunar surface (see Figure 3a; Dobrovolskis, 1981). The nearest material on the lunar surface is the closest local material at position $\boldsymbol{p}_{\boldsymbol{l}}$ at landing time of $\boldsymbol{t}_{\boldsymbol{l}}$. We track the movement of this local material and consider its final location as the final position of the ejecta hitting on this local material. This method has been used for the study of postemplacement effect of the ejecta in the modeling of large impact basins on the Moon (Zhu et al., 2015, 2017). The illustration in Figure 4 shows how we treated the sliding of ejecta once it lands on the Moon.

We calculate the ejecta thickness distribution along the distance from the impact site based on the final position of the ejecta with the effects of postemplacement motion. We divide the surrounding surface of the basin into discrete rings of 2-arc-degree. The ejecta thickness is calculated from the number of tracers (i.e., mass) located at a given distance from the point of impact up to an arc distance of antipode $\left(180^{\circ}\right)$ assuming the initial density of the material. It is likely that ejecta deposits contain a significant amount of porosity and we may underestimate the ejecta thickness as the assumed density may be too high. However, the given 

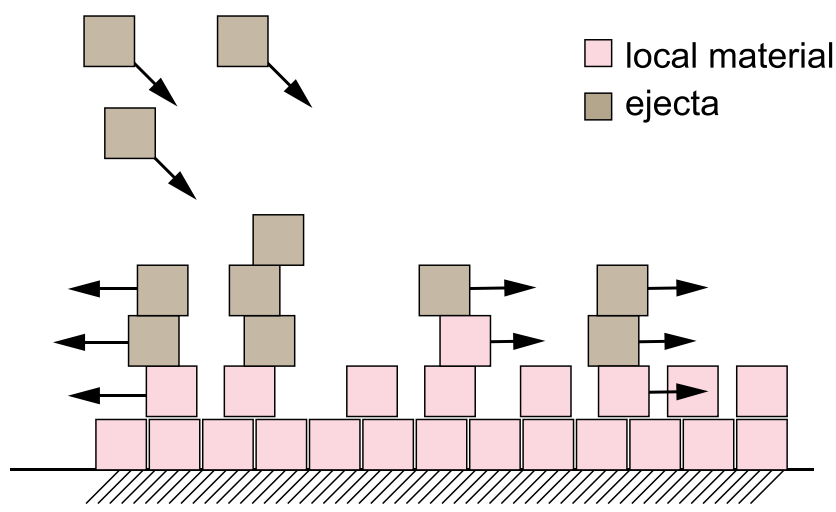

Figure 4. The carton shows the methods used for the ejecta sliding calculation and the structure of the new crust. The red blocks represent the local crust material over the surface of the Moon. The dark blocks represent the ejecta. The arrows show the directions of movements or the displacements of these blocks. When the ejecta lands on the local material, it follows the movement of the local material. The ejecta, as a new layer covering on the displaced crustal material, and the displaced crust form the postimpact crust. thickness of the ejecta deposits may cause self-compaction, which in turn reduces the porosity. To estimate the porosity of the ejecta deposit is currently not possible and we believe it is the best approach at the current state to assume the largest possible density, which corresponds to zero porosity. The approach to estimate the ejecta thickness as described has been validated against laboratory impact experiments into sand (Wünnemann et al., 2016), where the density problem is not an issue.

Simulations of the ejecta plume (e.g., Artemieva et al., 2013) indicate that the amount of material that is deposited on the ground as a consequence of collapse of the plume and the condensation of vaporized materials produces a very thin layer of dust particle and fine-grained ejecta mixed with the condensation products. We consider this layer to be negligible for the global-scale process we are interested in. For this reason and because modeling the fate of the ejecta plume is computationally very expensive we do not account for vaporization of material.

In our models, the postimpact crust is composed of two components: (1) the original preimpact crustal material and (2) ejecta deposited on top on the original crust. Before impact the original crust of the Moon is assumed to be $40 \mathrm{~km}$ thick. As mentioned above, the original crust may be displaced during the basin formation (e.g., crust around the basin rim may slump into the basin interior or flow outwards during the basin-forming process; see section 5.1), we calculate the displaced crustal thickness, similar to the determination of the ejecta thickness, by the number of displaced crustal tracers accumulating into discrete rings of 2-arc-degree width from impact site $\left(0^{\circ}\right)$ up to an arc distance of antipode $\left(180^{\circ}\right)$. As we assume ejecta that lands on the surface to be part of the new crust, the total thickness of the postimpact crust can be calculated by summing up the displaced crustal thickness and the postsliding ejecta thickness (see Figure 4). Note, as the ejecta include the material of impactor, and excavated crustal and mantle materials of the target, the ejected mantle materials deposited close to the basin rim that then slumped back into the basin interior are not included in our calculation for the thickness of new crust. It means that the postimpact crust within the basin interior only contains the crustal contribution.

\section{Results}

In total, we carried out 360 simulations of giant impacts to investigate whether such an event could reproduce the crustal thickness asymmetry and two-layer structure on the farside highlands. With the constrains of crustal thickness distribution from the model derived from GRAIL observations, we find that a range of simulations with different combinations of impact velocities and impactor size are in reasonable agreement with the observations. For example, the combinations of impactor diameter $\left(D_{\mathrm{imp}}\right)$ and impact velocity $\left(v_{\text {imp }}\right)$, such as $D_{\text {imp }}=500 \mathrm{~km}$ with $v_{\text {imp }}=15 \mathrm{~km} \mathrm{~km} / \mathrm{s}, D_{\text {imp }}=600 \mathrm{~km}$ with $v_{\text {imp }}=12 \mathrm{~km} / \mathrm{s}^{-1}, D_{\text {imp }}=720$ $\mathrm{km}$ with $v_{\text {imp }}=6.8 \mathrm{~km} / \mathrm{s}, D_{\text {imp }}=780 \mathrm{~km}$ with $v_{\text {imp }}=5.2 \mathrm{~km} / \mathrm{s}, D_{\text {imp }}=780 \mathrm{~km}$ with $v_{\text {imp }}=6.4 \mathrm{~km} / \mathrm{s}$, $D_{\text {imp }}=780 \mathrm{~km}$ with $v_{\text {imp }}=7.5 \mathrm{~km} / \mathrm{s}, D_{\text {imp }}=820 \mathrm{~km}$ with $v_{\text {imp }}=5.2 \mathrm{~km} / \mathrm{s}$, and $D_{\text {imp }}=820 \mathrm{~km}$ with $v_{\text {imp }}$ $=5.4 \mathrm{~km} / \mathrm{s}$ for a warm Moon (with the temperature profile TP2) reproduce the observed structures. In this section, we report the results of our best fit model with an impactor with $780 \mathrm{~km}$ in diameter (iron core diameter of $200 \mathrm{~km}$ ) and an impact velocity of $6.4 \mathrm{~km} / \mathrm{s}$, which could reproduce not only the observed crustal thickness distribution but also the two-layer structure on the farside highlands with an ejecta layer $\sim 5-10$ $\mathrm{km}$ thick on top, as observed. The case of an impactor with $720 \mathrm{~km}$ in diameter hitting on a warm Moon with a velocity of $6.8 \mathrm{~km} / \mathrm{s}$ also reproduces the observed structures, which is shown in the supporting information (see Figure S2) with other impact combinations (see Figure S3-S8).

\subsection{Giant Impact Cratering Process}

Figure 5 illustrates the basin-forming process and temperature distribution of the best fit model. During the basin-forming process, the impactor penetrates into the target, displacing and excavating target material; the floor of the excavated cavity is covered with a thick veneer of impactor and crustal material with a high 
(A)

(B)

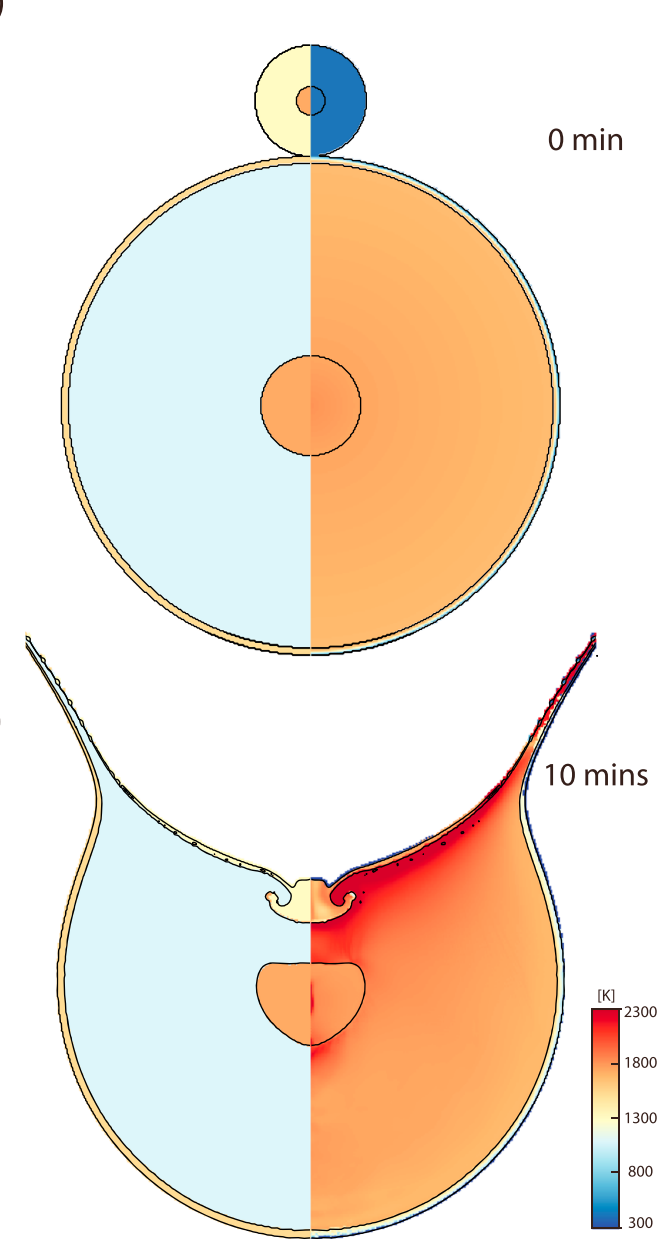

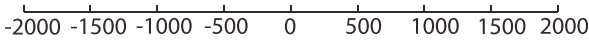

$(\mathrm{km})$
(C)

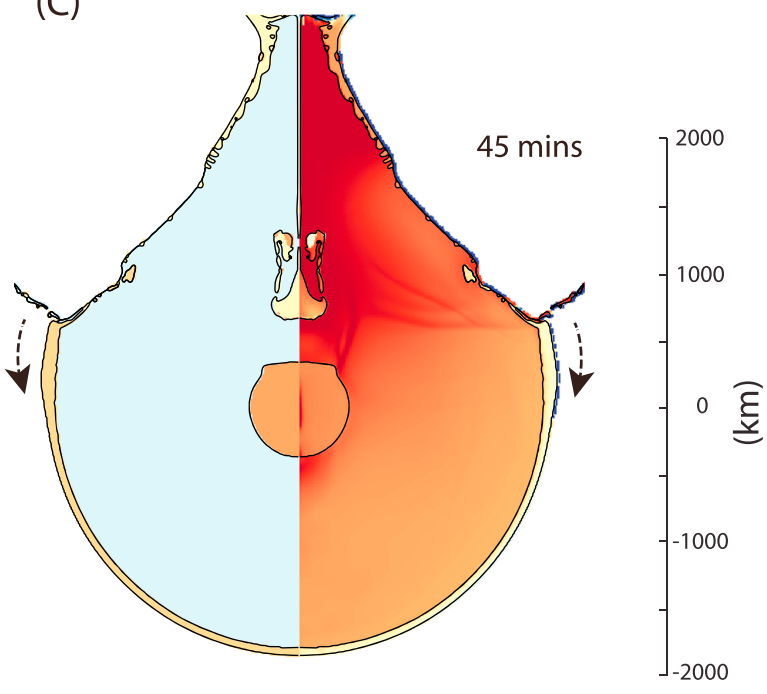

(D)

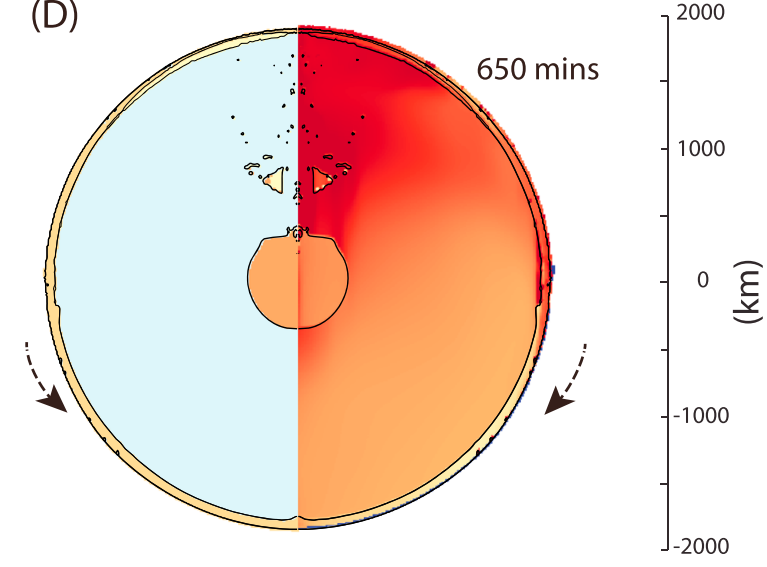

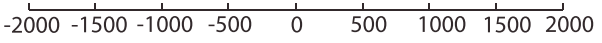

$(\mathrm{km})$

Figure 5. The basin-forming process for an impactor $780 \mathrm{~km}$ in diameter (200-km diameter of iron core) with an impact velocity of $6.4 \mathrm{~km} / \mathrm{s}$ using the warmer profile Thermal profile 2 (TP2). The Moon was assumed to be a 3,500-km-diameter sphere with 700-km-diameter of iron core. The crustal thickness is assumed to be $40 \mathrm{~km}$. In each panel, the left halves represent the materials used in the model: the gabbroic anorthosite (pale green), dunite (blue), and iron (orange) represent the lunar crust, mantle, and core, respectively. The gabbroic anorthosite (pale yellow) also represents the impactor material. The right halves represent the temperature variation during the impact process. The arrows in (c) and (d) represent the local crustal materials that were displaced and form the new crust together with deposited ejecta.

temperature (Figure $5 \mathrm{~b}$ ). While the cavity grows, the crater starts to collapse and form a central peak several hundred kilometers high. The outward material flow, formed during the collapse of the central uplift, drags crustal material adjacent to the crater rim into a bulge, giving rise to a thickened crustal region comparable to the farside highlands (Figure 5c, dashed arrows). Subsequent inward motion of hot material around the basin fills up the excavated cavity, forming a melt pool in the inner basin $(\sim 2,800 \mathrm{~km}$ in radius; see section 5.2$)$. This inward flow transports proximal ejecta back into the basin, erasing typical crater morphological features, such as the elevated rim and thick ejecta blanket adjacent to the rim (Figure 5d). During the impact process, the impactor's mantle partly mixes with the Moon's crustal material and remains near the surface inside the basin; most of the impactor's iron core $(>85 \%)$ mixes with the Moon's mantle beneath the impact site, and only a small fraction merges immediately with the Moon's core (see Figure 5d).

\subsection{Basin Size for the Best Fit Model}

The giant impact excavates materials down to a depth of $\sim 300 \mathrm{~km}$ around the impact site (Figure $6 \mathrm{a}$ ). The transient crater is defined as the cavity that results from the shock wave-induced excavation flow and 


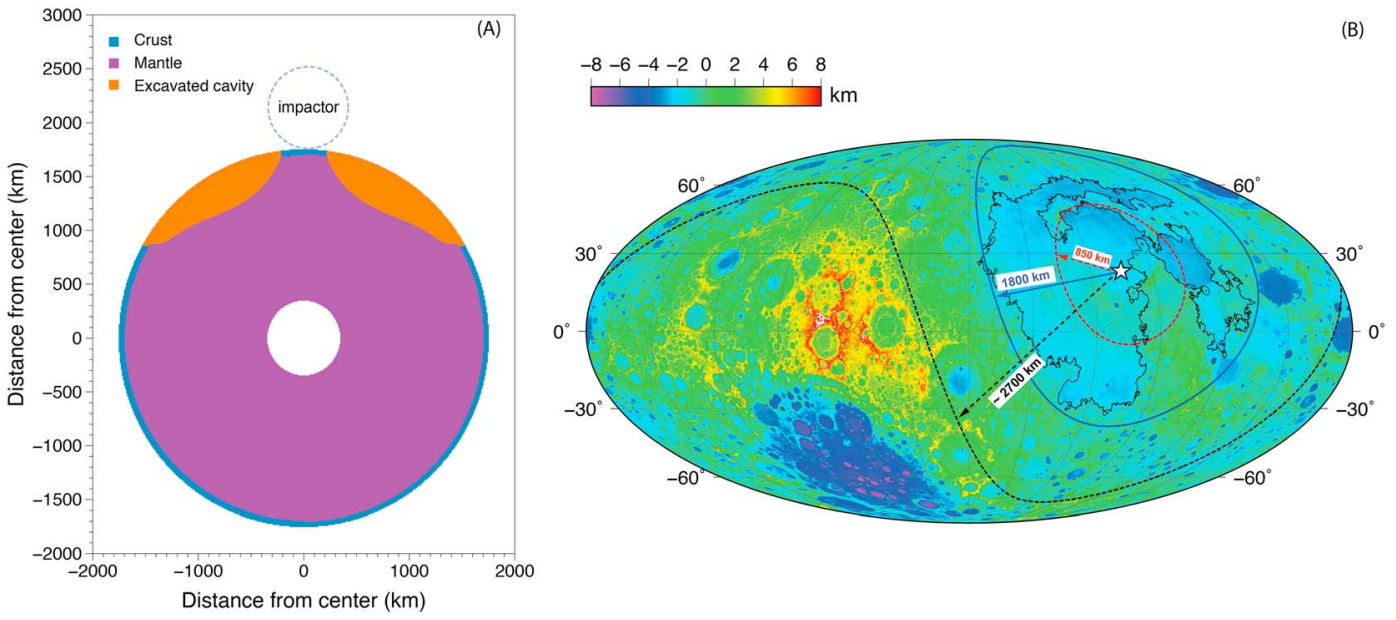

Figure 6. (a) The ejected material provenance for the giant impact event (780-km diameter impactor with velocity of 6.4 $\mathrm{km} / \mathrm{s}$ ). The crustal thickness was assumed to be $40 \mathrm{~km}$. The excavation cavity is the orange zone. The radius of the excavation cavity is the radial distance along the surface from the impact site to the boundary of excavation cavity. The dashed circle represents the size of impactor. (b) The boundary of transient (solid line) and final crater (black dashed line) for the giant impact event from this work; the transient crater with a radius of $850 \mathrm{~km}$ for the proposed Procellarum basin is also plotted as the red dashed circle for the comparison (Wilhelms, 1987). Data are presented in Mollweide equalarea projection centered at $90^{\circ} \mathrm{W}$ longitude, with nearside on the right of center and farside on the left of center. The background is the topographic distribution observed by Lunar Orbiter Laser Altimeter (Smith et al., 2010). The star on the nearside represents the assumed impact site.

marks the end of the so-called excavation stage and the beginning of the modification stage during the impact cratering process (e.g., Melosh, 1989). In particular, for basin-sized crater the transient crater does not reflect a certain time in the course of crater formation but is reached at different place of the transient cavity at different time. For instance, while the gravity-driven collapse occurs at the deepest point of the cavity, the crater continues to grow in diameter. For this reason, it is not straight forward to measure the dimension of the transient crater and different methods have been proposed. Elbeshausen et al. (2009) uses the first maximum in crater volume to approximate the transient at a certain point in time. Here we are more interested in the diameter of the transient crater and follow an approach by Spudis (1993), where the diameter of the transient crater is given by the radial distance from where material is expelled. We note that estimates of transient crater diameter by both definitions approaches to be equivalent for small craters. However, for the best fit model of the proposed giant impact, the transient crater radius, defined as the radial distance along the curved surface for the excavation cavity, is $\sim 1,800 \mathrm{~km}$ (see Figure 6a), in comparable to $\sim 2,000 \mathrm{~km}$ estimated by the method of maximum crater cavity (Elbeshausen et al., 2009).

Due to the scale of impact and the thermal conditions considered in this work, the definition of basin rim for the final crater diameter using traditional methods (i.e., a topographic rim) is not trivial. Here we propose the basin size as the radial distance along the surface from the impact site to a point where more than $90 \%$ of the ejecta experience very little (less than a cell size of $10 \mathrm{~km}$ ) inward sliding after the emplacement within the model time. This definition is plausible to represent the rim of large-scale basins without well-resolved topographic surface expression. The ejecta within the rim slide inward significantly owing to the collapse of the basin walls, whereas most of the ejecta deposited at the rim or further out do not move after landing and thus are not involved in the gravity-driven crater modification process. For the best fit model, the estimated radius of the final basin is $\sim 2,700-2,800 \mathrm{~km}$. Assuming that the impact site is at $15^{\circ} \mathrm{N}, 23^{\circ} \mathrm{W}$, the center of the Procellarum basin (Whitaker, 1981; Wilhelms, 1987; Wilhelms \& McCauley, 1971), Figure 6b shows the boundary of the modeled transient crater $(\sim 1,800$ $\mathrm{km})$ and final crater $(\sim 2,700 \mathrm{~km})$, from which we find that the boundary of the transient crater is consistent with the extent of the Procellarum KREEP Terrain (Jolliff et al., 2000), whereas the rim of the final basin $(\sim 2,700 \mathrm{~km})$ is located at the boundary between the farside and nearside. The basin size is far greater than the proposed Procellarum basin $(\sim 3,200 \mathrm{~km}$ diameter, Whitaker, 1981), but similar to the mega basin proposed on the nearside of the Moon (Byrne, 2007). 


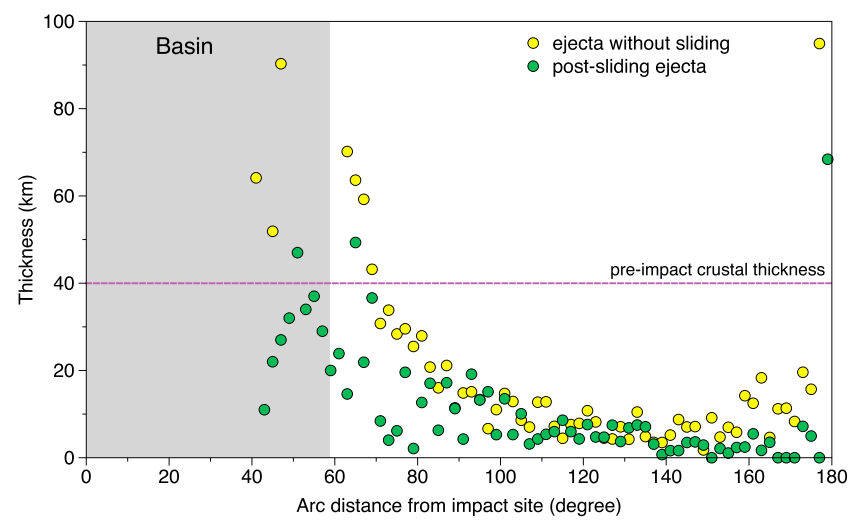

Figure 7. The ejecta thickness distribution before and after the consideration of ejecta sliding. The gray area represents the extent of the transient cavity. Within the basin (0-90 arc distance), we exclude the mantle material in the postsliding ejecta.

\subsection{Postimpact Ejecta Thickness Distribution}

The impact velocity of $6.4 \mathrm{~km} / \mathrm{s}$ in our best fit model matches well with the velocities of early impacts on the Moon (Marchi et al., 2012). The low impact velocity results in low ejection velocities, which leads to a relatively small amount of ejecta deposited on the farside highlands. Figure 7 illustrates the ejecta thickness variation along the radial distance from the impact site before (yellow points) and after (green points) the consideration of ejecta sliding for the best fit model. The ejecta thickness distribution is determined from the impact site $\left(0^{\circ}\right)$ up to an arc distance of the antipode $\left(180^{\circ}\right)$ with each interval of 2-arc-degree width. For the ejecta without considering sliding, the impact produces an ejecta layer more than $110 \mathrm{~km}$ at the transient crater rim (yellow points), which decreases to a thickness of $\sim 11 \mathrm{~km}$ at an arc distance of $100^{\circ}$ from the basin center. The ejecta thickness is $5-10 \mathrm{~km}$ at an arc distance of $170^{\circ}$ but increases rapidly to $\sim 90 \mathrm{~km}$ at the antipode of the impact site, at which the ejecta significantly piles up due to the small area for the 2-arcdegree width.

Assuming that the ejecta, once deposited on the surface, is further transported by material flow, we track this postexcavation movement to obtain the final location of excavated material. The sliding of ejecta changes the material around the rim of the basin (see green points). This is because ejecta near the rim of the basin are carried back into the basin cavity during the modification stage of basin formation (see Figure 7). When the ejected mantle material that flows back into the basin cavity, it was finally mixed with the local mantle material. The ejected crustal material also flows back into the basin cavity and mixes with local mantle material; however, it will subsequently float to the surface because of its lower density. Note, for the postsliding ejecta thickness calculation, we do not include the mantle ejecta that flowed back into the cavity. For example, the sharp decrease of the postsliding ejecta thickness (green points) within $40-90^{\circ}$ (the boundary of final crater) in Figure 7 is because we excluded the ejected mantle material that flowed back into the cavity. However, the decrease of ejecta around the antipode is due to the movement of ejecta in the direction of the impact site where the area of discrete rings is large, resulting in a decrease of the ejecta thickness. The postsliding ejecta layer is $\sim 30 \mathrm{~km}$ thick at the transient crater rim, which decreases to a thickness of $10 \mathrm{~km}$ at an arc distance of $100^{\circ}$, but retains $5-10 \mathrm{~km}$ at an arc distance to $170^{\circ}$ from the basin center, corresponding to the farside of the Moon. These ejecta, even partially mixed with the original crust once deposited on the lunar surface, still overlay the original crust, forming the layered crustal structure on the farside highlands with a thickness of $\sim 5-10 \mathrm{~km}$ for the top layer as proposed from the remote sensing observations (Donaldson Hanna et al., 2014; Yamamoto et al., 2012).

\subsection{Giant Impact Reproduces the Observed Crustal Thickness Asymmetry 5.4.1. The Modeled Crustal Thickness}

The modeled crustal thickness (see Figure 8, orange line), composed of the displaced crust (blue dashed line) and ejecta (green points), varies as a consequence of the outward motion of crust during the collapse of the central uplift (Figure 5c). It increases from $\sim 30 \mathrm{~km}$ at the transient crater boundary to $\sim 50 \mathrm{~km}$ at an arc distance of $90^{\circ}$ and reaches a maximum of $\sim 60 \mathrm{~km}$ at an arc distance of $140^{\circ}$, then decreases to $\sim 57 \mathrm{~km}$ at an arc distance of $180^{\circ}$. Assuming the impact site is at $15^{\circ} \mathrm{N}, 23^{\circ} \mathrm{W}$, the center of the proposed mega basin on the nearside of the Moon (Byrne, 2007; Whitaker, 1981; Wilhelms, 1987; Wilhelms \& McCauley, 1971), Figure 9a shows the global distribution of the modeled crustal thickness.

\subsubsection{Comparison With the Crustal Thickness Distribution Derived From GRAIL Observation}

The Moon experienced a long-term impact and volcanism history after the postgiant impact, and these activities were thought to have changed the localized crustal thickness distribution; however, the average profile of the observed crustal thickness (Figure 9b, Wieczorek et al., 2013) will not be significantly changed, therefore, can be directly used to compare with the modeled result. Figure $9 \mathrm{c}$ shows the comparison of the average profiles derived from the modeled crustal thickness (orange, from Figure 9a) and GRAIL gravity data (black, from Figure 9b), varied with the radial distance from the impact center. From Figure 9c, we can find that the giant impact can reproduce the average profile well from the arc 


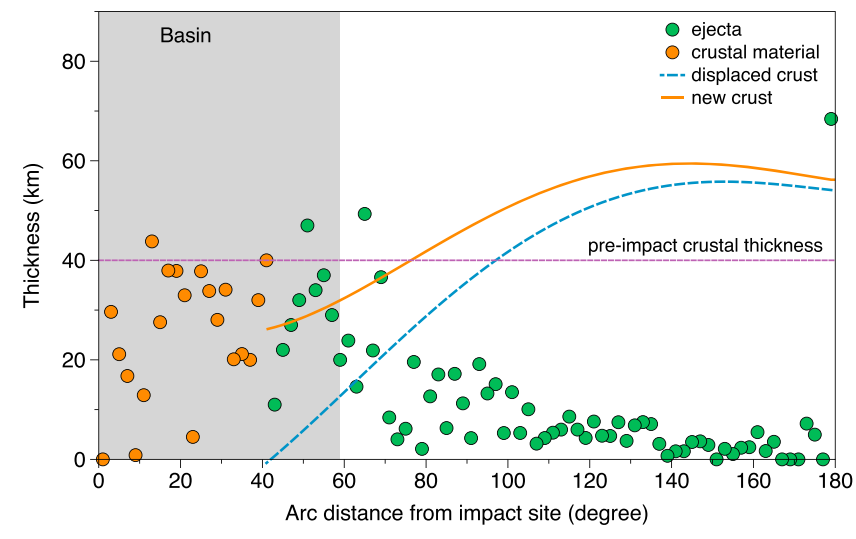

Figure 8. The ejecta and crustal thickness after postexcavation movement for the impact of a 780-km diameter impactor at $6.4 \mathrm{~km} / \mathrm{s}$. The impact site is at $0^{\circ}$. The gray section from 0 to $60^{\circ}$ represents the size of the transient crater (see Figure 6). Within the transient crater, the $\sim 25$ - to $30-\mathrm{km}$-thick crust (average) includes the deposited (orange points) and excavated crustal material that underwent postemplacement transport (green points within 40-60 degrees). The impactor and ejecta thickness distribution are binned into discrete rings of 2-arc degree width from the impact site up to an arc distance of $180^{\circ}$. The orange line represents the impact-derived crustal thickness composed of the displaced crust (blue dashed line) and ejecta (green points), starting from $40^{\circ}$ from the impact site. The blue dashed line represents the displaced crust that is originally adjacent to the crater rim and then was dragged by the outward material flow during the impact cratering process. distance of $60^{\circ}$ (the boundary of transient crater) to $150^{\circ}$ (the outer boundary of the SPA basin) within the standard errors. However, the giant impact produces the crustal thickness that reaches a high value in a ring between 120 and $180^{\circ}$ from the impact center, whereas the observations show a pronounced crustal thickness between 120 to $150^{\circ}$ We attribute this deviation to the SPA basin with a diameter of $\sim 2,500$ $\mathrm{km}$ on the farside (see Figure 9a), which is not considered in our simulation. The SPA basin was thought to have excavated a large amount of the originally thick crust beyond $150^{\circ}$ and formed a relatively thin crust (Potter et al., 2012), as observed.

We also use the swathed profiles of the crustal thickness to compare the variation of the localized crustal thickening centered on the farside highlands between the modeled crustal thickness and the crustal thickness derived from the GRAIL gravity data. Swathed profiles, the average data profiles from swaths centered at the farside highlands in the north, northeast, and east directions, can be described by the degree-two harmonics, which was thought to best represent the mean crustal profiles of the Moon and avoid the influences from the other geologic activities (e.g., subsequent impact events and volcanism; see the details in Garrick-Bethell et al., 2010). For each swath, the profile is calculated from transects obtained in two opposite (fit and prediction) directions. The profiles in fit directions were used to check the agreement of the modeled crustal thickness and observed crustal thickness. The profiles in prediction directions are used to test the subsequent effects on the variations of localized crustal thickness on the farside highlands. Table 2 lists the parameters of four swaths used in this work, in which $\varphi$ represents the azimuth of the great circle; $\theta$ and $\psi$ represents the swath width from the original points (longitude and latitude). Each swathed profile is calculated by averaging data profile between two great circle transects emanating from the latitude and longitude. For example, for the fit direction, the azimuths of the first and second great circle transect are defined by the angles of $\varphi$ and $\varphi+\theta$, separately. The mean profile of the crustal thickness in the fit direction is calculated by averaging great circle transects between $\varphi$ and $\varphi+\theta$, with each transect having an angular separation of $1^{\circ}$. Similarly, the prediction profile is calculated by averaging the data profiles between $\varphi+180$ and $\varphi+\theta+180$. Figure $9 \mathrm{~b}$ shows these swaths plotted on the global crustal thickness distribution derived from the GRAIL mission (Wieczorek et al., 2013).

We plot four swath profiles from the modeled crustal thickness, together with those derived from GRAIL gravity data (Wieczorek et al., 2013; see Figure 9d). In Figure 9d, the profiles in each panel correspond to the swathed profiles derived from the modeled crustal thickness (Figure 9a) and GRAIL gravity data (Figure 9b), from which we can find that the modeled crustal thickness reproduces the observed profiles at the farside highlands to an arc distance of $90-105^{\circ}$ reasonably well (fit region, represented by the solid green line; correlation coefficient $\left.R^{2}>0.96\right)$. Deviations occur at further distances $\left(>90^{\circ}\right)$ likely because of effects from subsequent large basin-forming impacts (e.g., Imbrium) that were not considered in this work. In the other direction $\left(<0^{\circ}\right)$, the modeled crustal-thickness profiles reproduce the observed profiles at the farside highlands to an arc distance of $-20^{\circ}$ well, but deviations occur at arc distance from -20 to $-50^{\circ}$, in which the observed crustal thickness is much lower than the model predicts (the dashed green line). For swath profiles 3 and 4, these deviations can be attributed to the SPA basin on the farside (see Figure 9a), which should have excavated a large amount of crustal material (Potter et al., 2012). However, the deviations in swath profiles 1 and 2, which have less affect by the SPA and other basins, imply a slightly broad region of the thicker crust predicted on the farside highlands by our impact model, which can be reconciled by a different-sized impactor.

In summary, our results broadly agree with the observed profiles and therefore can reproduce the variation of mean crustal profiles of the Moon, which suggests that the giant impact is a possible scenario for the formation of farside highlands and asymmetric crustal-thickness distribution of the Moon. 
(A) $\begin{array}{llllllll}25 & 30 & 35 & 40 & 45 & 50 & 55 & 60\end{array}$
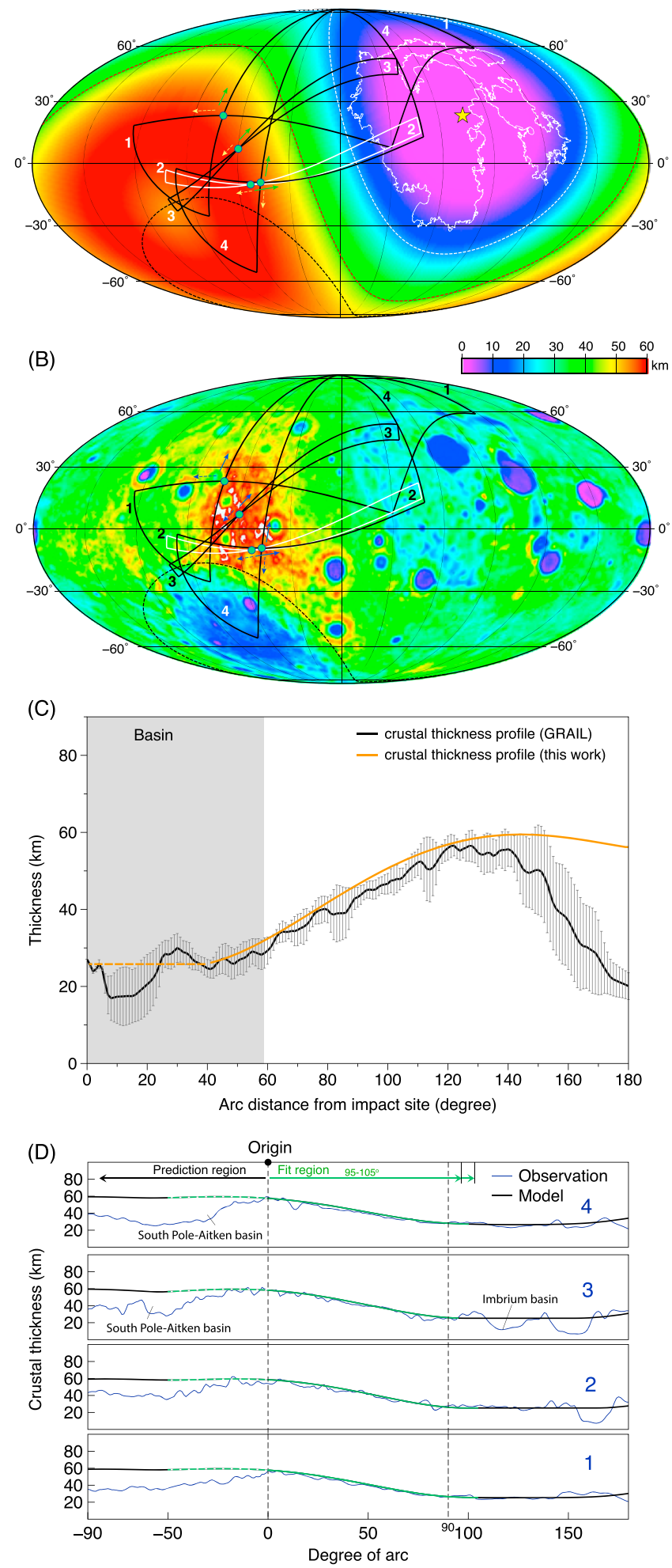
Table 2

Swath Dimensions From Garrick-Bethell et al (2010)

\begin{tabular}{llllll}
\hline Swath & Lat. ${ }^{\circ} \mathrm{N}$ & Lon. ${ }^{\circ} \mathrm{E}$ & $\varphi$ & $\theta$ & $\psi$ \\
\hline 1 & 23 & 198 & 0 & 90 & 100 \\
2 & -10 & 217 & 80 & 10 & 105 \\
3 & 7 & 210 & 40 & 10 & 95 \\
4 & -9 & 223 & 0 & 90 & 100 \\
\hline
\end{tabular}

\section{Discussion}

\subsection{Impact Melt Forming the Lowlands on the Nearside?}

As a consequence of shock compression and the subsequent unloading, large amounts of crustal material, as well as the projectile and some parts of the mantle, undergo melting during the impact cratering process. To quantify the melt production, we record the peak shock pressures the material experiences during the passage of shock wave using tracer particles. For the crustal material (gabbro), we assume, for simplicity, the critical peak shock pressures of $56 \mathrm{GPa}$ (Stöffler, 1972) and $91 \mathrm{GPa}$ for the softening (the onset of melt) and fully melting and neglect their dependences on the initial temperature because the temperature is low within the crust (Zhu et al., 2017). However, for the mantle material, we consider the target's temperature, lithostatic pressure, and density to estimate the critical pressure larger than the solidus and liquidus at different depth. We calculate the postshock temperatures by relaxing the material to the ambient pressure at the final location of each tracer (Pierazzo et al., 1997). Tracers with postshock temperatures between the solidus and liquidus were considered to be partially molten, with the fraction of melt varying linearly from zero at the solidus to one at the liquidus (Potter et al., 2013; Zhu et al., 2015). For example, the temperatures of 1,373 and 2,160 $\mathrm{K}$ are considered in our calculation for the softening and melting temperature of the dunite (Pierazzo et al., 1997; Zhu et al., 2017).

The giant impact produces partially molten material (melt fraction $>0$ ) with a total volume of $\sim 1.6 \times 10^{10}$ $\mathrm{km}^{3}$, where $\sim 4.5 \times 10^{9} \mathrm{~km}^{3}$ are totally molten (melt fraction =1). This molten material extends almost entirely over the impact hemisphere (see Figure 10a). Material with a melt fraction $>0.1$ reaches down to the core and extends radially to a distance of 1,600 km (point $\boldsymbol{b}$ in Figure 10a), corresponding to the size of mare basalt region (Jolliff et al., 2000) and the putative Procellarum basin (Wilhelms \& McCauley, 1971) on the nearside (see Figure 1a and Figure 10b). According to the modeling of the solidification of impact melt pool within the large impact basins, like Orientale and SPA (Hurwitz \& Kring, 2014; Vaughan et al., 2013; Vaughan \& Head, 2014), the impact melt pool will eventually cool and contract on a much longer time scale of 1-10 Ma (Schwinger \& Zhu, 2018), forming a depression with the similar size of the melt pool. Therefore, it is reasonable to predict that the melt pool produced by the giant impact would form a depression of the same size as the lowlands covering the Moon's nearside (see Figure 10b) when it eventually cools and contracts. Late-forming impact basins (e.g., Imbrium) can also form a melt pool but cannot form a depression the same size as the lowlands on the nearside.

Figure 9. (a) map of modeled crustal thickness distribution from the giant impact event; (b) the crustal thickness from Gravity Recovery and Interior Laboratory (GRAIL) observations (Wieczorek et al., 2013); (c) the comparison of the average crustal-thickness profiles from the modeled crustal thickness (a) and that derived from GRAIL observations (b); (d) the comparison of the average crustal-thickness profiles for swath 1 to 4 derived from both the modeled crustal thickness (a) and the crustal thickness derived from GRAIL observation (b). For (a) and (b), data are presented in Mollweide equal-area projection centered at $90^{\circ} \mathrm{W}$ longitude, with nearside on the right of center and farside on the left of center. The solid and dashed arrows along each swath represent the fit and prediction direction as shown in Table 2. Points on the swaths represent the centers. In (a), the yellow star represents the impact center and the thin white line represents the Procellarum KREEP Terrane boundary on the nearside; the black dashed line represents the South PoleAitken (SPA) terrain; the white dashed line represents the size of transient crater $(r \sim 1,800 \mathrm{~km})$; the red dashed lines represents the final crater $(r \sim 2,800 \mathrm{~km})$; the solid and dashed arrows along each swath represent the fit and prediction direction, respectively. In (c), the gray section from 0 to $60^{\circ}$ represents the size of the transient crater; the error bars represent the standard errors for the average crustal-thickens profile. In (d), the thick black and blue lines (from -90 to $180^{\circ}$ ) represent the mean profiles of swaths derived from the modeled crustal thickness (a) and observed crustal thickness from GRAIL (b), respectively; the green lines (from -50 to $95^{\circ}$ or $105^{\circ}$ ) represent the mean profiles of swaths plotted on (a) and the solid green lines (from 0 to $95^{\circ}$ or $105^{\circ}$ ) represent mean profiles for the fit region. 
(A)

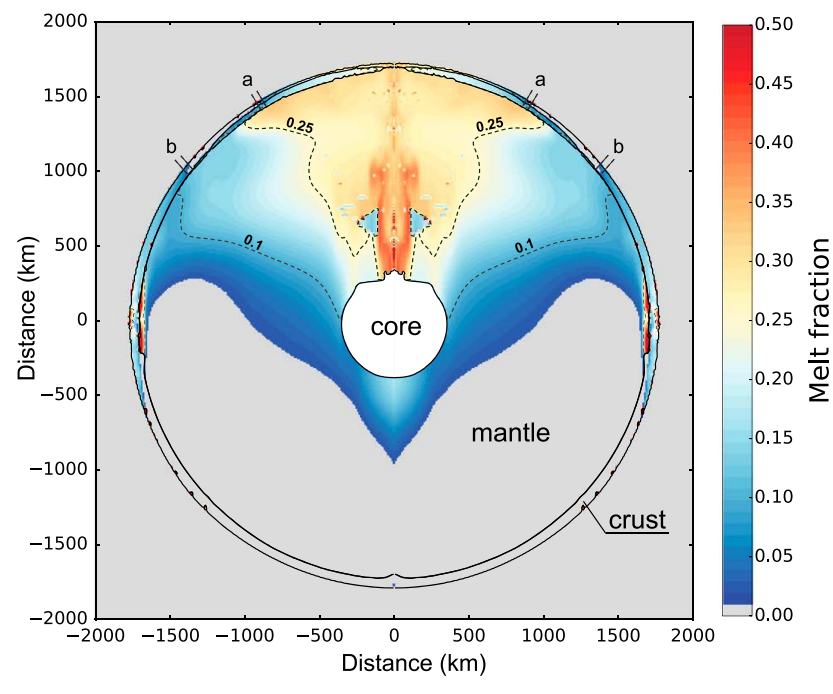

(B)

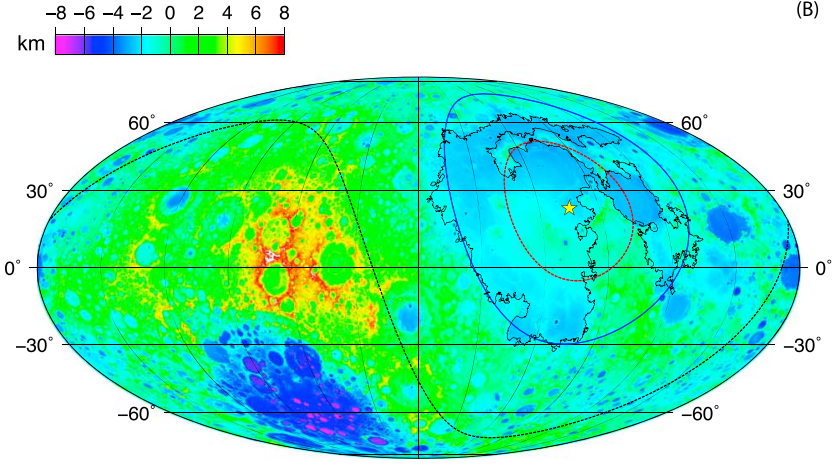

Figure 10. (a) The melt distribution of the giant impact event. Colored sections represent the molten material; gray sections represent unmolten material (melt fraction of 0 ). The contours represent the melt fraction with 0.1 and 0.25 . Point $\boldsymbol{a}$ and $\boldsymbol{b}$ indicates the boundary of the molten and unmolten material of the new crust. The radial distance is $\sim 900 \mathrm{~km}$ for point $\boldsymbol{a}$ and $\sim 1,600 \mathrm{~km}$ for point $\boldsymbol{b}$ from the impact site. The material of the new crust from point $\boldsymbol{a}$ to $\boldsymbol{b}$ is not molten. The complete molten material (melt fraction $\sim 1.0$ ) is located around the boundary of the basin, less than $0.1 \%$ of the total of the melt volume. (b) The topographic map of the Moon (leftfarside, right-nearside). The black line represents the outline of mare on the nearside. The red dashed and solid blue circle, with a radius of 900 and $1,600 \mathrm{~km}$, represents the boundaries of the molten and unmolten material of the new crust in A (point $\boldsymbol{a}$ and $\boldsymbol{b}$ ), respectively; the black dashed line represents the boundary of final basin from the modeling. The star on the nearside represents the impact site.
Within the basin, the impact melt rocks are mainly composed of target crustal (with some mantle) and impactor mantle rocks, to a radial distance of $\sim 900 \mathrm{~km}$ (point $\boldsymbol{a}$ in Figure 10a). Within this molten zone, the mixture of molten crustal material with mantle material supports the occurrence of olivine and low-calcium pyroxene that likely formed by an impact event (Nakamura et al., 2012). Farther out, up to a radial distance of 1,600 km (point $\boldsymbol{b}$ in Figure 10a), the crust does not undergo melting ( $\sim 0$ melt fraction), but beyond this distance and up to the boundary of the basin $(\sim 2,800 \mathrm{~km}$ from impact site), the crust is melted again $(\sim 0.1$ melt fraction).

Such a giant impact delivered a large amount of energy to the Moon. Rolf et al. (2017) studied the thermal and heat flux anomalies of the Moon as enhanced by a similar sized impact and found that the heat flux anomaly induced by the giant impact lasts only $100 \mathrm{Myr}$. However, according to their study, the giant impact can extract more basalts from the mantle within the basin on a long timescale (e.g., 1 Ga; see Rolf et al., 2017), which may contribute to the formation of the majority of mare basalts observed on the nearside of the Moon.

\subsection{Postimpact KREEP Material Distribution on the Lunar Surface}

According to the magma ocean model (Warren, 1985), the KREEP material was thought to be concentrated in the last magmas to crystallize and sandwiched between the crust and the mantle. From the remote sensing observations, the KREEP is found predominately on the nearside, but rarely on the farside highlands (Lawrence et al., 1998; Prettyman et al., 2006; Zhu et al., 2013). Several scenarios, including the asymmetric distribution of KREEP material (Hess and Parmentier, 2001), inhomogeneous differentiation of the magma ocean (Loper \& Werner, 2002), and opposite impact ejecta of a companion moon (Jutzi \& Asphaug, 2011), have been proposed to explain the asymmetric distribution of KREEP material on the Moon. However, its formation is still enigmatic.

In our model, the upper $20 \mathrm{~km}$ of mantle is assumed to represent a KREEP layer (Hess \& Parmentier, 1995) and its location was tracked throughout basin formation. During the basin formation, part of KREEP, at the impact site, is ejected along with crust and mantle material. The ejected KREEP that is deposited close to the basin slumps back into the basin, accumulating at the bottom of, or mixed into, the new crust (see Figure 11). The other part of KREEP at the impact site is compacted and mixed within the mantle, which may result in the subsequent magmatism widespread on the lunar nearside. In addition, the thick crust around the mega-basin produces heavy stress for the underneath KREEP layer, which may result in the lateral transport, to some extent, and accumulate the KREEP material within the basin (Mange \& Arkani-Hamed, 1991).

Due to the asymmetric crustal thickness after the giant impact, the depth of the KREEP layer varies between the nearside $(\sim 25-30 \mathrm{~km})$ and farside $(\sim 60-65 \mathrm{~km})$. Consequently, the relatively shallow KREEP layer at the nearside could have been excavated and transported more easily to the surface by subsequent basin-forming events (e.g., Imbrium), forming the KREEP distribution with high concentrations on the nearside and low concentrations on the farside (Lawrence et al., 1998; Prettyman et al., 2006). On the farside, significant impacts would be required to excavate KREEP or bring it closer to the surface from its 60- to $65-\mathrm{km}$ depth. For example, the Orientale basin, with a diameter of $960 \mathrm{~km}$ (see Figure 1), excavated material from a depth of $55 \mathrm{~km}$ (Zhu et al., 2015). 


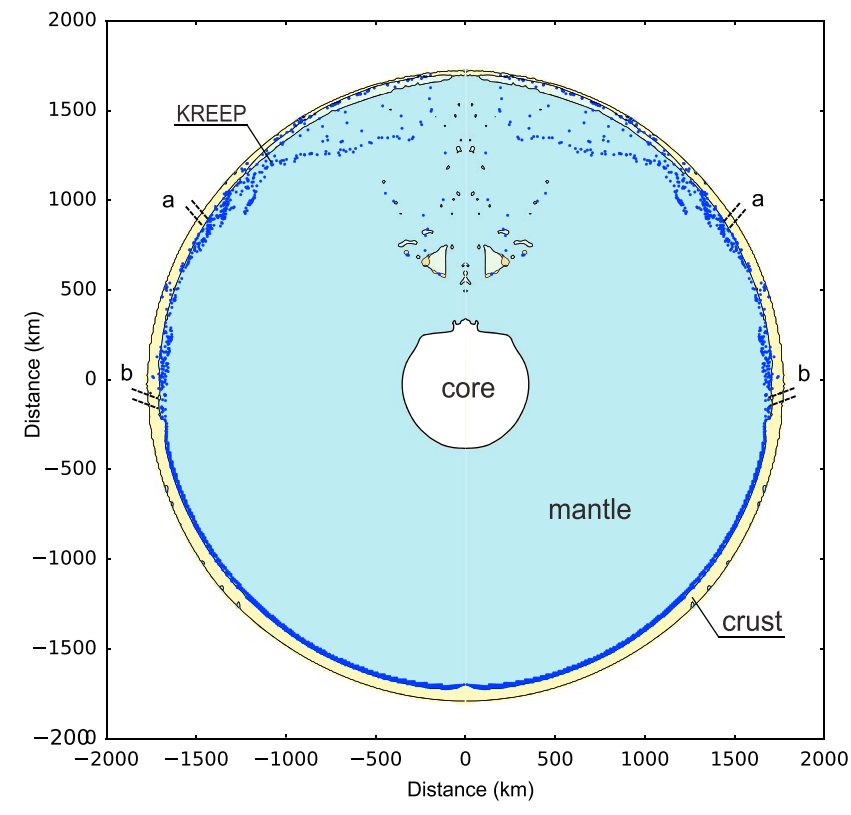

Figure 11. The postimpact KREEP material distribution. For our model, the global KREEP material was initially resided at the bottom of crust with a thickness of $20 \mathrm{~km}$. The giant impact excavated the crustal, KREEP, and mantle material. The ejected KREEP material, deposited close to the basin rim, slumped back into the basin and was emplaced below crust. Point $\boldsymbol{a}$ and $\boldsymbol{b}$ represent the boundaries of transient crater $(\sim 1,800 \mathrm{~km})$ and final crater $(\sim 2,700 \mathrm{~km})$ from the impact site. See Figure 6 for the boundaries of transient crater and final crater on the global crustal thickness map.
For the ejecta deposited on the farside highlands, $\sim 90 \%$ originates from the upper mantle, in which $\sim 10 \%$ represent the KREEP. Taking an $\sim 11 \mathrm{ppm}$ thorium concentration in the vicinity of Imbrium basin rim (Prettyman et al., 2006) as a proxy to represent the KREEP abundance underneath the new crust, the ejecta deposited on the farside highlands have $\sim 1$ ppm of thorium (10\% of ejected KREEP). After the giant impact event, subsequent large impacts and impact gardening on the farside highlands (e.g., Huang et al., 2017) may have further diluted the thorium concentration to $<1 \mathrm{ppm}$, in agreement with the remote sensing observations (Prettyman et al., 2006). For example, SPA, the largest basin on the farside, was thought to be formed after the proposed giant impact. Melosh et al. (2017; as with Potter et al., 2012) argues that the SPAforming impact may have excavated the Moon up to a depth of $\sim 100 \mathrm{~km}$ and produced an ejecta layer with a thickness of several hundreds of meters on the farside highlands. The components of the SPA ejecta deposited on the farside highlands include the lunar crust, mantle, and KREEP materials, with a thorium concentration $<1 \mathrm{ppm}$ (estimated from Figure 2 in Melosh et al., 2017). These ejecta should have mixed and diluted the thorium concentration on the farside highlands produced by the giant impact.

However, we have to note that the initial KREEP concentration underneath the crust before the giant impact is unknown. It is possible that the KREEP may not be evenly globally concentrated underneath the crust at the formation of the giant impact (e.g., Laneuville et al., 2013). This is based on the remote sensing observations that the thorium concentration in the vicinity of Imbrium basin ( $>10 \mathrm{ppm})$ in the PKT is greater than the value around the Apollo basin ( 2 ppm) in the SPA (see Figure 1c, Prettyman et al., 2006). The Apollo basin, $\sim 500 \mathrm{~km}$ in diameter, should have excavated mantle and, thus also KREEP material (Potter et al., 2018). The low thorium concentration at Apollo basin can be only explained by the reduced concentration of KREEP beneath the SPA before its formation (e.g., Laneuville et al., 2018).

\subsection{Postimpact Compositions on the Farside Highlands}

Our model of a giant impact suggests that the postsliding ejecta with a thickness of 5-10 km on the farside may have formed the lunar highlands. According to our simulation, these ejecta are excavated and expelled from a depth down to $\sim 300 \mathrm{~km}$ around the impact site (Figure 6), in which the vast majority ( $\sim 90 \%)$ of the ejected materials originate from the upper mantle. These ejecta cover the primordial anorthositic crust and produce a much more mafic-rich layer, forming the two-layer structure on the farside highlands, as suggested by the compositional observations (e.g., Donaldson Hanna et al., 2014; Yamamoto et al., 2012). As the ejecta have high temperature $(>1,800 \mathrm{~K}$; see Figure 5$)$ and were melted to various degrees along the radial distance from the rim of the basin, they may have differentiated to some extent (Hurwitz \& Kring, 2014; Vaughan et al., 2013).

The composition of the upper mantle of the early Moon at the time of impact is not well known, and assumptions are based mostly on theoretical and indirect considerations. It has been proposed that the upper mantle of the early Moon is dominated by orthopyroxene rather than olivine (Lucey et al., 2014; Melosh et al., 2014; Melosh et al., 2017). This assumption is based on the relatively small abundance of olivine in the vicinity of large impact basins (e.g., the SPA basin) that undoubtedly must have excavated mantle material to the surface, spectral data, lunar samples, seismic, and petrological studies (Lucey et al., 2014; Melosh et al., 2014; Melosh et al., 2017; Wieczorek, 2006). An orthopyroxene-rich upper mantle is also supported by modeling of the thermal evolution of the LMO (Elkins-Tanton \& Bercovici, 2014; Khan et al., 2006; Kuskov \& Kronrod, 1998). Although the olivine has been observed around the Crisium and Moscoviense basins (Yamamoto et al., 2010), which may have penetrated through the lunar crust (Miljkovic et al., 2015; Wieczorek et al., 2013), the exposures of olivine is thought to originate most likely from the lower crust 
(Head \& Wilson, 1992; Pieters et al., 2011). As the distribution of olivine can be assumed to be heterogeneous laterally and vertically within the lower crust (Martinot, 2018), it cannot be the dominated material of upper mantle of the Moon.

Based on the compositional assumptions of the upper mantle, the proposed giant impact suggests that orthopyroxene-rich mantle material was ejected and deposited at the farside highlands, forming an ejecta layer with a thickness of $\sim 5-10 \mathrm{~km}$. According to our simulation, $\sim 90 \%$ of the ejected materials originate from the upper mantle, which is admittedly much higher than the estimated value of $\sim 20 \%$ from the remote sensing observations (Crites \& Lucey, 2015; Lucey et al., 2014). In addition, the ejecta would produce a much higher mafic concentration on the farside highlands than the observations indicated (e.g., $4-5$ wt.\% of FeO; see Lucey et al., 2014). However, the predicted high concentrations of pyroxene and mafic material on the farside highlands do not mean the model results are incompatible with the observations. This is because the proposed giant impact event occurred at an early stage of the evolution of the Moon. After this event the Moon experienced a long-term bombardment history. As a consequence, the ejecta from the proposed impact on the farside highlands would have been covered by ejecta from subsequent giant impacts, such as the SPA basin (Hawke et al., 2003; Melosh et al., 2017; Yamamoto et al., 2015). Subsequent large-scale impact basins on the farside highlands (e.g., Birkhoff, Coulomb-Sarton, and Lorentz) would have penetrated through the ejecta layers and exposed the primordial crust to the surface, as it is observed (Donaldson Hanna et al., 2014; Matsunaga et al., 2009). The long-term impact gardening (i.e., Huang et al., 2017) would have mixed the material on the surface (due to the high degree of complexity this subsequent reworking of ejecta strata has not been considered in this work). These activities may eventually dilute the mafic concentration of materials on the surface of farside highlands to the present-day value (Lucey et al., 2014) and produce a relatively low concentration of pyroxene as observed (e.g.,Crites \& Lucey, 2015 ; Lucey et al., 2014).

The upper mantle of the Moon was thought to have a $\mathrm{Mg} \#(\mathrm{Mg} /(\mathrm{Mg}+\mathrm{Fe})$ in mole per cent) of $75-86$ to a depth of $270 \mathrm{~km}$, based on the modeling of the thermal evolution of the LMO (Elkins-Tanton \& Bercovici, 2014; Khan et al., 2006; Kuskov \& Kronrod, 1998; Wieczorek, 2006). The postimpact material on the farside highlands should have a similar Mg\#, as estimated from the remote sensing observations (e.g., Crites \& Lucey, 2015; Ohtake et al., 2012).

\subsection{Origin of the Impactor and Implications for the ${ }^{182} \mathrm{~W}$ Composition of the Moon}

An origin of the Moon's nearside-farside asymmetries by a giant impact implies a substantial addition of mass to the Moon subsequent to its formation. This raises the question of whether this additional mass left an imprint on the Moon's isotopic composition. In this section, we will address this question with a particular emphasis on the ${ }^{182} \mathrm{~W}$ composition of lunar samples, which is sensitive to the late addition of material to planetary mantles.

The Earth and Moon exhibit a striking isotopic similarity for several elements (e.g., O and Ti), which otherwise show large variations among solar system bodies (i.e., asteroids and Mars; Wiechert et al., 2001; Young et al., 2016; Zhang et al., 2012). These isotope anomalies are commonly used as genetic tracers, because they reflect a specific mixture of presolar and nebular components. As such, bodies that formed in different regions of the accretion disk are expected to exhibit distinct isotope compositions. It is therefore remarkable that the Earth and Moon exhibit indistinguishable isotope compositions, although most giant impact models predict that the Moon predominantly consists of impactor material, meaning that the isotopic composition of the Moon should reflect that of the impactor and not the Earth. One way to account for the isotopic homogeneity of the Earth and Moon is to assume that the Moon-forming impactor and proto-Earth both have formed from an isotopically homogeneous inner disk reservoir (Dauphas, 2017; Dauphas et al., 2014). It has also been proposed that the Moon predominantly consists of proto-Earth material (Canup, 2012; Cuk \& Stewart, 2012) or that there has been postgiant impact equilibration between the Earth and Moon (Lock et al., 2018; Pahlevan \& Stevenson, 2007).

In the two preferred models considered here, the nearside-farside asymmetry formed by impact of a differentiated body with a total mass of between $7.6 \times 10^{20} \mathrm{~kg}$ and $9.7 \times 10^{20} \mathrm{~kg}$ (diameter of between 720 and 780 $\mathrm{km}$ ). Our model shows that this material would be mixed with a total mass of lunar material of $\sim 3 \times 10^{21}$ $\mathrm{kg}$ and thus contributed $\sim 20-25 \%$ of mass to the final mixture between impactor and lunar material. As the proposed impact would have affected a large area on the Moon's nearside, including all Apollo 


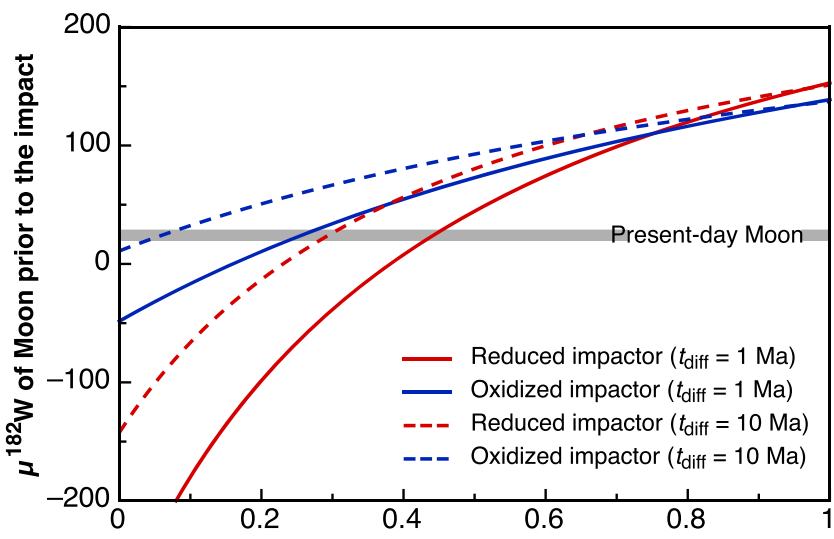

Fraction of projectile core equilibrated with target mantle

Figure 12. The predicted $\mu^{182} \mathrm{~W}$ of the Moon prior to impact and effects for different impactor compositions and different differentiation timescales after solar system formation. The grey horizontal bar represents the $\mu^{182} \mathrm{~W}$ $=24 \pm 5$ ppm for the Moon (Kruijer et al., 2015; Touboul et al., 2015). Calculations assume a mass of the impactor $7.6 \times 10^{20} \mathrm{~kg}$ and that the impactor mixed with a mass of $3 \times 10^{21} \mathrm{~kg}$ of lunar mantle material (within the volume of $\sim 25 \%$ melt fraction; see Figure 10a). Assumed mantle W concentration were $19 \mathrm{ppb}$ (Moon) and 5 (reduced, red lines) and 55 (oxidized, blue lines) ppb in the impactor mantle (see the details in the main text). landing sites, it is conceivable that all samples collected at these sites have the isotopic composition of the mixture between the original lunar material and material derived from the asymmetry-forming impactor. The isotopic composition of the Apollo samples, therefore, provides information on the origin of this impactor and on the overall effect of the impact on the Moon's isotopic composition.

The indistinguishable isotopic compositions of the Earth and Moon, as derived from the Apollo lunar samples, imply that the asymmetryforming impactor had a very similar isotopic composition compared to the Earth and Moon; otherwise, the impact would have resulted in an isotopic anomaly in the Moon. For instance, ordinary chondrites, which presumably derive from S-type asteroids (one of the most common asteroid types in the inner solar system) are characterized by a ${ }^{50} \mathrm{Ti}$ anomaly of $\varepsilon^{50} \mathrm{Ti} \approx-0.7\left(\varepsilon^{50} \mathrm{Ti}\right.$ is the parts-per-10,000 deviation of the ${ }^{50} \mathrm{Ti} /{ }^{47} \mathrm{Ti}$ ratio relative to the terrestrial standard; Trinquier et al., 2009). Thus, if the asymmetry-forming impactor had an ordinary chondrite-like isotopic composition, then this would have changed the Ti isotopic composition of the Moon (i.e., the region of the Moon affected by the impact) to $\varepsilon^{50} \mathrm{Ti} \approx-0.15$ (assuming similar $\mathrm{Ti}$ concentrations for impactor and Moon). However, all lunar samples analyzed to date have a common $\varepsilon^{50} \mathrm{Ti}$ of $-0.03 \pm 0.04$, indistinguishable from the composition of the Earth (Zhang et al., 2012). Conversely, to not induce a ${ }^{50} \mathrm{Ti}$ anomaly in the Moon, the $\varepsilon^{50} \mathrm{Ti}$ anomaly of the asymmetry-forming impactor must have been within about \pm 0.25 of that of the Earth. The only known meteoritic materials with such small ${ }^{50} \mathrm{Ti}$ anomalies are enstatite chondrites and aubrites. Although these meteorites do not represent the building material of the Earth and Moon (Render et al., 2017), isotopically, they are the closest match and, as such, probably formed at a similar heliocentric distance as Earth's building blocks (Dauphas, 2014; 2017). The asymmetry-forming impactor, therefore, likely derived from the same region of the accretion disk as Earth's building material, including the Moon-forming impactor.

Whereas the asymmetry-forming impact likely did not induce an isotope anomaly in the Moon for elements like Ti and $\mathrm{O}$, for $\mathrm{W}$ isotopes the situation is different. This is because ${ }^{182} \mathrm{~W}$ variations do not result from the heterogeneous distribution of presolar and nebular components but reflect the chemical fractionation of $\mathrm{Hf}$ from $\mathrm{W}$ during planetary differentiation and subsequent decay of ${ }^{182} \mathrm{Hf}$ (half-life $=8.9 \mathrm{Ma}$; e.g., Kleine \& Walker, 2017). Variations in ${ }^{182} \mathrm{~W}$, therefore, provide a record of the accretion and differentiation history of planetary bodies and not of their genetic heritage. As the ${ }^{182} \mathrm{~W}$ composition of the bulk asymmetryforming impactor likely was chondritic and, hence, different from that of the lunar mantle, this impact likely induced a change in the ${ }^{182} \mathrm{~W}$ composition of the Moon.

We calculated the effect of the impact on the ${ }^{182} \mathrm{~W}$ composition of the lunar mantle, using different ${ }^{182} \mathrm{~W}$ compositions of the impactor's mantle and core. As the impactor composition and differentiation is not $a$ priori known, we assumed core formation ages between 1 and $10 \mathrm{Ma}$ after solar system formation. These timescales are typical for the differentiation of asteroids up to Mars-sized bodies (e.g., Kleine et al., 2009) and are, therefore, a reasonable estimate for the differentiation time of the asymmetry-forming impactor. The ${ }^{182} \mathrm{~W}$ compositions of this impactor's mantle and core not only depend on the time of differentiation but also on the Hf/W ratio of the mantle. This ratio is determined by the metal-silicate partition coefficient $D$ for W, which in turn primarily depends on the redox conditions during core formation. We, therefore, calculated the expected ${ }^{182} \mathrm{~W}$ compositions for both a 'reduced' $(D=100)$ and an 'oxidized' impactor $(D=5)$. Figure 12 shows the effects on the ${ }^{182} \mathrm{~W}$ composition of the lunar mantle (i.e., the area affected by the asymmetry-forming impact) for different impactor compositions and different timescales. The calculations show that this effect strongly depends on the extent to which the impactor core equilibrated with the lunar mantle and that depending on this effect, the ${ }^{182} \mathrm{~W}$ composition of the lunar mantle can either increase or decrease (here $\mu^{182} \mathrm{~W}$ is the part-per-million deviation from the present-day ${ }^{182} \mathrm{~W} /{ }^{184} \mathrm{~W}$ of the bulk silicate Earth). As our simulations suggest that a significant fraction $(>85 \%)$ of the impactor core equilibrated 
with the lunar mantle, the asymmetry-forming impact probably significantly lowered the ${ }^{182} \mathrm{~W}$ composition of that part of the lunar mantle that was affected by this impact. Consequently, the preimpact ${ }^{182} \mathrm{~W}$ composition of the Moon might have been between $\sim 100$ and $\sim 150 \mu^{182} \mathrm{~W}$ (see Figure 12), implying that prior to the asymmetry-forming impact, the Moon might have had an ${ }^{182} \mathrm{~W}$ anomaly relative to Earth's mantle.

The finding that the Moon originally might have had an ${ }^{182} \mathrm{~W}$ anomaly potentially has important implications for understanding the origin of the Moon. Analyses on lunar samples reveal that the Moon exhibits an $\sim 25 \mathrm{ppm}{ }^{182} \mathrm{~W}$ excess over the present-day bulk silicate Earth (Kruijer et al., 2015; Kruijer \& Kleine, 2017; Touboul et al., 2015). This ${ }^{182} \mathrm{~W}$ excess is thought to entirely reflect disproportional late accretion to the Earth and Moon. The term 'late accretion' (or late veneer) is commonly used to describe the addition of primitive, broadly chondritic material to the mantles of the Earth and Moon after the Moon-forming impact and the cessation of HSE sequestration into the core (by either metal-silicate differentiation or FeS exsolution during LMO crystallization and mantle overturn). The amount of late-accreted material, as estimated from HSE abundances inferred for each mantle, is much lower for the Moon ( 0.02 wt.\%) compared to the Earth (0.5-0.8 wt.\%; Day \& Walker, 2015). Given this large difference in late-accreted material, mass balance calculations predict that the ${ }^{182} \mathrm{~W}$ composition of the bulk silicate Earth was lowered by $\sim 25 \mathrm{ppm}$, whereas the lunar ${ }^{182} \mathrm{~W}$ was not changed significantly. As such, the $\sim 25 \mathrm{ppm}{ }^{182} \mathrm{~W}$ difference between the present-day bulk silicate Earth and the Moon can entirely be accounted for by disproportional late accretion (Kruijer et al., 2015; Kruijer \& Kleine, 2017; Touboul et al., 2015). Consequently, these observations suggest that immediately following the Moon-forming impact, and prior to late accretion, the Earth's mantle and the Moon had indistinguishable ${ }^{182} \mathrm{~W}$ compositions.

However, the ${ }^{182} \mathrm{~W}$ similarity observed between the prelate veneer Earth's mantle and the Moon is unexpected in the canonical giant-impact model of lunar origin, which predicts a significant ${ }^{182} \mathrm{~W}$ excess in the Moon (Kruijer \& Kleine, 2017). This conclusion holds regardless of the amount of impactor material in the Moon, meaning that unlike for other isotope systems, the Earth-Moon similarity for ${ }^{182} \mathrm{~W}$ cannot easily be accounted for by making the Moon out of proto-Earth's mantle material. The ${ }^{182} \mathrm{~W}$ data, therefore, suggest that postgiant processes modified the ${ }^{182} \mathrm{~W}$ composition of the Moon, either through EarthMoon equilibration (Lock et al., 2018) or as shown in the present study, by the asymmetryforming impact.

\section{Further Work}

It has been argued that most basins on the Moon were formed by the oblique impact. Therefore, a threedimensional modeling approach using the 3-D iSALE code (Elbeshausen et al., 2009) would be the logical approach for future studies on the formation of the proposed giant impact event and its ejecta distribution. An oblique impact may result in an elliptical crater and an asymmetric crustal thickness in the up-range and the down range direction, depending on the impact angle.

Currently, iSALE 2-D provides greater functionality and can be run at much higher resolution than its 3-D counterpart, thus offering important and detailed insights into basin formation and ejecta distribution. In our simulation, we approximate the gravity field to be static and radially symmetric with the origin at the center of the Moon. Apparently, during the formation of the proposed basin of the given size, the mass distribution temporarily deviates significantly from radial symmetry, and therefore, the gravity field needs to be adjusted dynamically according to the varying mass distribution. Taking the so-called self-gravity into account would be realistic for the simulation of a giant impact but is computationally very expensive. The computational demand for systematic study, which requires hundreds of simulations, is beyond the computer resources available for this study. However, we consider the proposed impact scenario to be small that self-gravity may be negligible and the gravity field can be considered to be static (central gravity). To test the effect of self-gravity on the cratering process and ejecta emplacement, we compare the early stages of basin formation, when mass distribution deviates most from radial symmetry due to the excavation of the transient crater, for a 2-D vertical impact model with self-gravity and central gravity (see Figure S1 in the supporting information). The models are almost indistinguishable and, therefore, we assume that the central gravity simplification as used in this study produces the same ejecta layer on the farside highlands as if selfgravity is considered. 
The 2-D central gravity simulations of vertical impacts in this study are a significant simplification neglecting the effect of obliquity of the impact and the dynamically changing gravity field. However, previous modeling studies (e.g., Hood \& Artemieva, 2008; Shuvalov, 2011) and a simple test comparing crater formation in a dynamically varying gravity field (self-gravity) with a static gravity field (central gravity) show that inside one crater radius, which includes the farside highlands, the ejecta distribution only weakly depends on the impact angle and that self-gravity does not influence at least the early stages of crater formation and ejecta deposition. Although, consider our models as a first-order estimate and late stages processes, such as the gravity-driven crater modification resulting in the final basin morphology, may be more significantly affected by impact angle and self-gravity. However, the given systematic parameters study provides important constraints on the potential formation of the lunar lowlands and highlands as a consequence of a giant impact. More sophisticated 3-D self-gravity studies for the proposed impact dimensions are intended as a logical next step, which will be possible upon further code developments of iSALE-3-D.

Finally, as the composition of the Moon's upper mantle is still puzzling and little is known for sure about the composition and structure of the lunar farside highlands, further studies are required to investigate the composition of the upper mantle and its relationship to the composition on the farside highlands of the Moon.

\section{Conclusions}

Our results demonstrate, for the first time, that a giant impact on the Moon, like that on Mars (Marinova et al., 2008), could explain the observed asymmetries in crustal thickness, elevation, and surface mineralogy, as the model results match well with observations; the lowlands on the nearside may be a consequence of this giant impact. However, such an impact would produce a basin with a diameter of 5,600 km, covering $\sim 50 \%$ of the Moon, far greater than the putative Procellarum basin with a diameter of 3,200 km. Yet the typical surface expression of this mega basin was erased due to the higher internal temperatures and thus, lower viscosity, of the young planet, resulting in the observed palimpsest-like basins (Bottke et al., 2015; Miljkovic et al., 2017) and a weak basin-like gravity signature (Andrews-Hanna et al., 2014; Miljkovic et al., 2017).

\section{Acknowledgments}

We greatly appreciate reviews from the Editor Steven A. Hauck, Elizabeth Silber, and two anonymous reviewers for insightful reviews that improved the quality of the manuscript. We also thank Jeffery Taylor and anonymous reviewers for valuable comments of the early versions. We thank the developers of iSALE (www.isale-code.de). Tom Davison developed the pySALEPlot tool used in this work. C. Brennecka provided assistance during the paper preparation. The GRAIL crustal thickness data can be accessed online via the GRAIL Crustal Thickness Archive (http://www.ipgp.fr/ wieczor/) The parameters for the iSALE model to reproduce this work are included and illustrated in manuscript. All data to reproduce the figures in this work can be accessed online (https://doi.org/ 10.5281/zenodo.2644673). This work was funded by the Deutsche Forschungsgemeinschaft (SFB-TRR 170), publication no. 5. M. Z. is supported by the Science and Technology Development Fund of Macau (079/2018/A2 and 043/2016/A2). R. W. K. P was supported by NASA Solar System Exploration Research Virtual Institute, SEEED (SSERVI Evolution and Environment of Exploration Destinations) cooperative agreement NNA14AB01A at Brown University.
Our model suggests that a significant fraction of the projectile core equilibrated with the mantle. As such, the asymmetry-forming impact may have lowered the $\mu^{182} \mathrm{~W}$ value of a large area of the Moon, which would include all of the Apollo landing sites. If more than $\sim 85 \%$ of the impactor core equilibrated within the lunar mantle, the preimpact ${ }^{182} \mathrm{~W}$ composition of the Moon would have been between $\sim 100$ and $\sim 150 \mu^{182} \mathrm{~W}$, implying that prior to the asymmetry-forming impact, the Moon might have had an ${ }^{182} \mathrm{~W}$ anomaly relative to immediate postgiant impact Earth's mantle, as expected in the giant impact model of lunar origin. This model suggests that there might be areas on the lunar farside whose ${ }^{182} \mathrm{~W}$ compositions were less strongly affected. As such, this model might be testable by ${ }^{182} \mathrm{~W}$ measurements on lunar meteorites, some of which probably derive from the Moon's farside.

\section{References}

Ahrens, T. J., \& O'Keefe, J. D. (1977). Equations of state and impact induced shock-wave attenuation on the Moon. In D. J. Roddy, R. O. Peppin, \& R. B. Merrill (Eds.), Impact and Explosion Cratering, (pp. 639-656). New York: Pergamon.

Andrews-Hanna, J. C., Besserer, J., Head III, J. W., Howett, C. J. A., Kiefer, W. S., Lucey, P. J., et al. (2014). Structure and evolution of the lunar Procellarum region as revealed by GRAIL gravity data. Nature, 514(7520), 68-71. https://doi.org/10.1038/nature13697

Artemieva, N. A., \& Shuvalov, V. V. (2008). Numerical simulation of high-velocity impact ejecta following falls of comets and asteroids onto the Moon. Solar System Research, 42(4), 329-334. https://doi.org/10.1134/S0038094608040059

Artemieva, N. A., Wünnemann, K., Krien, F., Reimold, W. U., \& Stöffler, D. (2013). Ries crater and suevite revisited-Observation and modeling. Part II: Modeling. Meteoritics and Planetary Science, 48(4), 590-627. https://doi.org/10.1111/maps.12085

Benz, W., Cameron, A. G. W., \& Melosh, H. J. (1989). The origin of the Moon and the single-impact hypothesis III. Icarus, 81(1), 113-131. https://doi.org/10.1016/0019-1035(89)90129-2

Besserer, J., Nimmo, F., Wieczorek, M. A., Weber, R. C., Kiefer, W. S., McGovern, P. J., et al. (2014). GRAIL gravity constraints on the vertical and lateral density structure of the lunar crust. Geophysical Research Letters, 41, 5771-5777. https://doi.org/10.1002/ 2014GL060240

Bottke, W., et al. (2005). The fossilized size distribution of the main asteroid belt. Icarus, 175(1), 111-140. https://doi.org/10.1016/j. icarus.2004.10.026

Bottke, W. F., Walker, R. J., Day, J. M. D., Nesvorny, D., \& Elkins-Tanton, L. (2010). Stochastic late accretion to Earth, the Moon, and Mars. Science, 330(6010), 1527-1530. https://doi.org/10.1126/science.1196874

Bottke, W. F. et al. (2015). The earliest lunar bombardment produced by Moon-forming impact ejecta. Early Solar System Impact Bombardment III, \#3012. 
Byrne, C. J. (2007). A large basin on the near side of the Moon. Earth, Moon and Planets, 101(3-4), 153-188. https://doi.org/10.1007/s11038007-9225-8

Canup, R. M. (2012). Forming a Moon with an Earth-like composition via a giant impact. Science, 338(6110), 1052-1055. https://doi.org/ $10.1126 /$ science. 1226073

Collins, G., et al. (2011). The size-frequency distribution of elliptical impact crater. Earth and Planetary Science Letters, 310(1-2), 1-8. https://doi.org/10.1016/j.epsl.2011.07.023

Collins, G. S., Melosh, H. J., \& Ivanov, B. A. (2004). Modeling damage and deformation in impact simulations. Meteoritics and Planetary Science, 39(2), 217-231. https://doi.org/10.1111/j.1945-5100.2004.tb00337.x

Crites, S. T., \& Lucey, P. G. (2015). Revised mineral and Mg\# maps of the Moon from integrating results from the Lunar Prospector neutron and gamma-ray spectrometers with Clementine spectroscop. American Mineralogist, 100, 937-982.

Cuk, M., \& Stewart, S. T. (2012). Making the Moon from a fast-spinning Earth: A giant impact followed by resonant despinning. Science, 338(6110), 1047-1052. https://doi.org/10.1126/science.1225542

Dauphas, N. (2017). The isotopic nature of the Earth's accreting material through time. Nature, 541(7638), 521-524. https://doi.org/ 10.1038 /nature20830

Dauphas, N., Burkhardt, C., Warren, P. H., \& Fang-Zhen, T. (2014). Geochemical arguments for an Earth-like Moon-forming impactor. Philosophical Transactions of the Royal Society A, 372(2024), 20130244. https://doi.org/10.1098/rsta.2013.0244

Day, J. M. D., Pearson, D. G., \& Taylor, L. A. (2007). Highly siderophile element constraints on accretion and differentiation of the EarthMoon system. Science, 315(5809), 217-219. https://doi.org/10.1126/science.1133355

Day, J. M. D., \& Walker, R. J. (2015). Highly siderophile depletion in the Moon. Earth and Planetary Science Letters, 423, 114-124. https:// doi.org/10.1016/j.epsl.2015.05.001

Day, J. M. D., Walker, R. J., James, O. B., \& Puchtel, I. S. (2010). Osmium isotope and highly Siderophile element systematics of the lunar crust. Earth and Planetary Science Letters, 289(3-4), 595-605. https://doi.org/10.1016/j.epsl.2009.12.001

Dobrovolskis, A. (1981). Ejecta patterns diagnostic of planetary rotations. Icarus, 47(2), 203-219. https://doi.org/10.1016/00191035(81)90167-6

Donaldson Hanna, K. L., Cheek, L. C., Pieters, C. M., Mustard, J. F., Greenhagen, B. T., Thomas, I. R., \& Bowles, N. E. (2014). Global assessment of pure crystalline plagioclase across the Moon and implications for the evolution of the primary crust. Journal of Geophysical Research: Planets, 119, 1516-1545. https://doi.org/10.1002/2013JE004476

Elbeshausen, D., Wnnemann, K., \& Collins, G. S. (2009). Scaling of oblique impacts in frictional targets: Implications for crater size and formation mechanisms. Icarus, 204, 716-731. https://doi.org/10.1016/j.icarus.2009.07.018

Elbeshausen, D., Wünnemann, K., \& Collins, G. S. (2013). The transition from circular to elliptical impact crater. Journal of Geophysical Research: PLanets, 118, 2295-2309. https://doi.org/10.1002/2013JE004477

Elkins-Tanton, L., Burgess, S., \& Yin, Q. Z. (2011). The lunar magma ocean: Reconciling the solidification process with lunar petrology and geochronology. Earth and Planetary Science Letters, 304(3-4), 326-336. https://doi.org/10.1016/j.epsl.2011.02.004

Elkins-Tanton, L. T., \& Bercovici, D. (2014). Contraction or expansion of the Moon's crust during magma ocean freezing? Philosophical Transactions of the Royal Society A, 372(2024), 20130240. https://doi.org/10.1098/rsta.2013.0240

Freed, A. M., et al. (2014). The formation of lunar mascon basins from impact to contemporary form. Journal Geophysical Research, 119, 2378-2397. https://doi.org/10.1002/2014je004657

Garrick-Bethell, I., Nimmo, F., \& Wieczorek, M. A. (2010). Structure and formation of the lunar farside highlands. Science, 330(6006), 949-951. https://doi.org/10.1126/science.1193424

Ghods, A., \& Arkani-Hamed, J. (2007). Impact-induced convection as the main mechanism for formation of lunar mare basalts. Journal of Geophysical Research, 112, E03005. https://doi.org/10.1029/2006je002709

Grimm, R. E. (2013). Geophysical constraints on the lunar Procellarum KREEP Terrane. Journal of Geophysical Research:Planets, 118, 768-777. https://doi.org/10.1029/1999je001092

Hawke, B. R., et al. (2003). Distribution and modes of occurrence of lunar anorthosite. Journal of Geophysical Research, 108(E6), 5050. https://doi.org/10.1029/2002JE001890

Head, J. W., \& Wilson, L. (1992). Lunar mare volcanism: stratigraphy, eruption conditions, and the evolution of secondary crusts. Geochimica et Cosmochimica Acta, 56(6), 2155-2175. https://doi.org/10.1016/0016-7037(92)90183-J

Hess, P. C. (1994). Petrogenesis of lunar troctolites. Journal of Geophysical Research, 99(E9), 19083-10,093. https://doi.org/10.1029/94JE01868

Hess, P. C., \& Parmentier, E. M. (1995). A model for the thermal and chemical evolution of the Moon's interior: Implications for the onset of mare volcanism. Earth and Planetary Science Letters, 134(3-4), 501-514. https://doi.org/10.1016/0012-821X(95)00138-3

Hess, P. C., \& Parmentier, E. M. (2001). Thermal evolution of a thicker KREEP liquid layer. Journal of Geophysical Research, 106(E11), 28,023-28,032. https://doi.org/10.1029/2000JE001416

Hood, L. L., \& Artemieva, N. A. (2008). Antipodal effects of lunar basin-forming impacts: Initial 3D simulations and comparisons with observations. Icarus, 193(2), 485-502. https://doi.org/10.1016/j.icarus.2007.08.023

Hörz, F., Ostertag, R., \& Rainey, D. A. (1983). Bunte Breccia of the Ries: Continuous deposits of large impact crater. Reviews of Geophysics and Space Physics, 21(1), 667-1,725.

Huang, Y.-H., Minton, D. A., Hirabayashi, M., et al. (2017). Heterogeneous impact transport on the Moon. Journal of Geophysical Research: Planets, 122, 1158-1180. https://doi.org/10.1002/2016je005160

Hurwitz, D. M., \& Kring, D. A. (2014). Differentiation of the South Pole-Aitken basin impact melt sheet: Implications for lunar exploration Journal of Geophysical Research: Planets, 119, 1110-1133. https://doi.org/10.1002/2013je004530

Ivanov, B. A., et al. (2010). Basin-forming impacts: Reconnaissance modeling. In R. L. Gibson, \& W. U. Reimold (Eds.), Large Meteorite Impacts and Planetary Evolution IV, (pp. 29-49). (Geological Society of America, Boulder, CO).

Jolliff, B. L., et al. (2000). Major lunar crustal terranes: Surface expressions and crust-mantle origins. Journal of Geophysical Research, 105, 4197-4216.

Jutzi, M., \& Asphaug, E. (2011). Forming the lunar farside highlands by accretion of a companion moon. Nature, 476(7358), 69-72. https:// doi.org/10.1038/nature10289

Khan, A., Maclennan, J., Taylor, S. R., \& Connolly, J. A. D. (2006). Are the Earth and the Moon compositionally alike? Inferences on lunar composition and implications for lunar origin and evolution from geophysical modeling. Journal of Geophysical Research, 111, E05005. https://doi.org/10.1029/2005je002608

Kleine, T., Touboul, M., Bourdon, B., Nimmo, F., Mezger, K., Palme, H., et al. (2009). Hf-W chronology of the accretion and early evolution of asteroids and terrestrial planets. Geochimica et Cosmochimica Acta, 73(17), 5150-5188. https://doi.org/10.1016/j. gca.2008.11.047 
Kleine, T., \& Walker, R. J. (2017). Tungsten isotopes in planets. Annual Review of Earth and Planetary Sciences, 45(1), 389-417. https://doi. org/10.1146/annurev-earth-063016-020037

Krasinsky, G. A., et al. (2002). Hidden mass in the asteroid belt. Icarus, 158, 98-105. https://doi.org/10.1006/icar.2002.6837

Kruijer, T. S., \& Kleine, T. (2017). Tungsten isotopes and the origin of the Moon. Earth and Planetary Science Letters, 475, 15-24. https://doi. org/10.1016/j.epsl.2017.07.021

Kruijer, T. S., Kleine, T., Fischer-Gödde, M., \& Sprung, P. (2015). Lunar tungsten isotopic evidence for the late veneer. Nature, 520(7548), 534-537. https://doi.org/10.1038/nature14360

Kuskov, O. L., \& Kronrod, V. A. (1998). Constitution of the Moon: 5. Constraints on composition, density, temperature, and radius of a core. Physics of the Earth and Planetary Interiors, 107(4), 285-306. https://doi.org/10.1016/S0031-9201(98)00082-X

Laneuville, M., Taylor, J., \& Wieczorek, M. (2018). Distribution of radioactive heat sources and thermal history of the Moon. Journal of Geophysical Research: Planets, 123, 3144-3166. https://doi.org/10.1029/2018JE005742

Laneuville, M., Wieczorek, M., \& Breuer, D. (2013). Asymmetric thermal evolution of the Moon. Journal of Geophysical Research: Planets, 118, 1435-1452. https://doi.org/10.1002/jgre.20103

Lawrence, D. J., et al. (1998). Global elemental maps of the Moon: the Lunar Prospector gamma ray spectrometer. Science, 281, 1484-1489.

Lee, D.-C., \& Halliday, A. N. (1997). Core formation on Mars and differentiated asteroids. Nature, 388(6645), 854-857. https://doi.org/ $10.1038 / 42206$

Lock, S. J., Stewart, S. T., Petaev, M. I., Leinhardt, Z., Mace, M. T., Jacobsen, S. B., \& Cuk, M. (2018). The origin of the Moon within a terrestrial synestia. Journal of Geophysical Research: Planets, 123, 910-951. https://doi.org/10.1002/2017JE005333

Loper, D. E., \& Werner, C. L. (2002). On lunar asymmetries 1. Tilted convection and crustal asymmetry. Journal of Geophysical Research, 107(E6), -5046. https://doi.org/10.1029/2000JE001441

Lucey, P. G., Blewett, D. T., \& Hawke, B. R. (1998). Mapping the $\mathrm{FeO}$ and $\mathrm{TiO}_{2}$ content of the lunar surface with multispectral imagery. Journal of Geophysical Research, 103(E2), 3679-3699. https://doi.org/10.1029/97JE03019

Lucey, P. G., Norman, J. A., Crites, S. T., Taylor, G. J., Hawke, B. R., Lemelin, M., \& Melosh, H. J. (2014). A large spectral survey of small lunar craters: Implications for the composition of the lunar mantle. American Mineralogist, 99(11-12), 2251-2257. https://doi.org/ 10.2138/am-2014-4854

Luther, R., Zhu, M. H., Collins, G., \& Wünnemann, K. (2018). Effect of target properties and impact velocity on ejecta dynamics and ejecta deposition. Meteoritics and Planetary Science, 53(8), 1705-1732. https://doi.org/10.1111/maps.13143

Mange, M., \& Arkani-Hamed, J. (1991). Remelting mechanisms for shallow source regions of mare basalts. Physics of the Earth and Planetary Interiors, 68(1-2), 9-31. https://doi.org/10.1016/0031-9201(91)90003-Z

Marchi, S., Bottke, W. F., Elkins-Tanton, L. T., Bierhaus, M., Wuennemann, K., Morbidelli, A., \& Kring, D. A. (2014). Widespread mixing and burial of Earth's Hadean crust by asteroid impacts. Nature, 511(7511), 578-582. https://doi.org/10.1038/nature13539

Marchi, S., Canup, R. M., \& Walker, R. J. (2018). Heterogeneous delivery of silicate and metal to the Earth by large planetesimals. Nature Geoscience, 11(1), 77-81. https://doi.org/10.1038/s41561-017-0022-3

Marchi, S., et al. (2012). The onset of the lunar cataclysm as recorded in its ancient crater populations. Earth and Planetary Science Letters, $325,27-38$.

Marinova, M. M., Aharonson, O., \& Asphaug, E. (2008). Mega-impact formation of the Mars hemispheric dichotomy. Nature, 453(7199), 1216-1219. https://doi.org/10.1038/nature07070

Martinot, M., et al. (2018). Compositional variations in the vicinity of the lunar crust-mantle interface from Moon mineralogy mapper data Journal of Geophysical Research: Planets, 123, 3220-3237. https://doi.org/10.1029/2018je005744

Melosh, H. J. (1989). Impact cratering: A geologic process, (p. 245). Oxford University Press.

Melosh, H. J., Freed, A. M., Johnson, B. C., Blair, D. M., Andrews-Hanna, J. C., Neumann, G. A., et al. (2013). The origin of lunar mascon basins. Science, 340(6140), 1552-1555. https://doi.org/10.1126/science.1235768

Melosh, H. J., Kendall, J., Horgan, B., Johnson, B. C., Bowling, T., Lucey, P. G., \& Taylor, G. J. (2017). South-Pole-Aitken basin ejecta reveal the Moon's upper mantle. Geology, 45(12), 1063-1066. https://doi.org/10.1130/G39375.1

Melosh, H. J. et al. (2014). The Moon's upper mantle: mostly OPX, not olivine? Lunar Planet. Sci. Conf., \#2505.

Milbury, C., Johnson, B. C., Melosh, H. J., Collins, G. S., Blair, D. M., Soderblom, J. M., et al. (2015). Preimpact porosity controls the gravity signature of lunar craters. Geophysical Research Letters, 42, 9711-9716. https://doi.org/10.1002/2015GL066198

Miljkovic, K., Wieczorek, M. A., Collins, G. S., Laneuville, M., Neumann, G. A., Melosh, H. J., et al. (2013). Asymmetric distribution of lunar impact basins caused by variations in target properties. Science, 342(6159), 724-726. https://doi.org/10.1126/science. 1243224

Miljkovic, K., et al. (2015). Excavation of the lunar mantle by basin-forming impact events on the Moon. Earth and Planetary Science Letters, 409, 243-251. https://doi.org/10.1016/j.epsl.2014.10.041

Miljkovic, K. et al. (2017). Elusive formation of impact basins on the young Moon. Lunar Planet. Sci., XLVIII, \#1361.

Morbidell, A., et al. (2018). The timeline of the Lunar bombardment-revisited. Icarus, 305, 262-276. https://doi.org/10.1016/j. icarus.2017.12.046

Morbidelli, A., et al. (2012). A sawtooth-like timeline for the first billion years of lunar bombardment. Earth and Planetary Science Letters, $355,144-151$.

Nakamura, R., Yamamoto, S., Matsunaga, T., Ishihara, Y., Morota, T., Hiroi, T., et al. (2012). Compositional evidence for an impact origin of the Moon's Procellarum basin. Nature Geoscience, 5(11), 775-778. https://doi.org/10.1038/ngeo1614

Neukum, G., \& Ivanov, B. A. (1994). Crater size distributions and impact probabilities on Earth from lunar, terrestrial planet, and asteroid cratering data. In T. Gehrels (Ed.), Hazards Due to Comets and Asteroids, (pp. 359-416). Tucson: University of Arizona Press.

Oberbeck, V. R. (1975). The role of ballistic erosion and sedimentation in lunar stratigraphy. Reviews of Geophysics, 13(2), 337-362. https:// doi.org/10.1029/RG013i002p00337

Ohtake, M., Matsunaga, T., Haruyama, J., Yokota, Y., Morota, T., Honda, C., et al. (2009). The global distribution of pure anorthosite on the Moon. Nature, 461(7261), 236-240. https://doi.org/10.1038/nature08317

Ohtake, M., Takeda, H., Matsunaga, T., Yokota, Y., Haruyama, J., Morota, T., et al. (2012). Asymmetric crustal growth on the Moon indicated by primitive farside highland materials. Nature Geoscience, 5(6), 384-388. https://doi.org/10.1038/ngeo1458

Pahlevan, K., \& Stevenson, D. J. (2007). Equilibration in the aftermath of the lunar-forming giant impact. Earth and Planetary Science Letters, 262(3-4), 438-449. https://doi.org/10.1016/j.epsl.2007.07.055

Parmentier, E. M., Zhong, S., \& Zuber, M. T. (2002). Gravitational differentiation due to initial chemical stratification: origin of lunar asymmetry by the creep of dense KREEP? Earth and Planetary Science Letters, 201(3-4), 473-480. https://doi.org/10.1016/S0012$821 \mathrm{X}(02) 00726-4$ 
Pierazzo, E., Vickery, A. M., \& Melosh, H. J. (1997). A reevaluation of impact melt product. Icarus, 127(2), 408-423. https://doi.org/10.1006/ icar.1997.5713

Pieters, C. M., Besse, S., Boardman, J., Buratti, B., Cheek, L., Clark, R. N., et al. (2011). Mg-spinel lithology: A new rock type on the lunar farside. Journal of Geophysical Research, 116, E00G08. https://doi.org/10.1029/2010JE003727

Potter, R. W., et al. (2015). Scaling of basin-sized impacts and the influence of target temperature. In V. G. R. Osinski, \& D. A. Kring (Eds.), Large Meteorite Impacts and Planetary Evoluiton, (Vol. 518, pp. 99-113). Special Paper.

Potter, R. W., et al. (2018). The Apollo peak-ring impact basin: Insights into the structure and evolution of the South Pole-Aitken basin. Icarus, 306, 139-149. https://doi.org/10.1016/j.icarus.2018.02.007

Potter, R. W. K., Collins, G. S., Kiefer, W. S., McGovern, P. J., \& Kring, D. A. (2012). Constraining the size of the South Pole-Aitken basin impact. Icarus, 220(2), 730-743. https://doi.org/10.1016/j.icarus.2012.05.032

Potter, R. W. K., et al. (2013). Numerical modeling of the formation and structure of the Orientale impact basin. Journal of Geophysical Research: Planets, 118, 1-17. https://doi.org/10.1002/jgre.20080

Prettyman, T. H., Hagerty, J. J., Elphic, R. C., Feldman, W. C., Lawrence, D. J., McKinney, G. W., \& Vaniman, D. T. (2006). Elemental composition of the lunar surface: analysis of gamma ray spectroscopy data from Lunar Prospector. Journal of Geophysical Research, 111, E12007. https://doi.org/10.1029/2005JE002656

Render, J., Fischer-Gödde, M., Burkhardt, C., \& Kleine, T. (2017). The cosmic molybdenum-neodymium isotope correlation and the building material of the Earth. Geochemical Perspectives Letters, 3, 170-178.

Rolf, T., Zhu, M. H., Wünnemann, K., \& Werner, S. C. (2017). The role of impact bombardment history in lunar evolution. Icarus, 286, 138-152. https://doi.org/10.1016/j.icarus.2016.10.007

Rubie, D. C., Laurenz, V., Jacobson, S. A., et al. (2016). Highly siderophile elements were stripped from Earth's mantle by iron sulfide segregation. Science, 353, 1141-1144.

Ryder, G. (2002). Mass flux in the ancient Earth-Moon system and benign implications for the origin of life on Earth. Journal of Geophysical Research, 107(E4), 5022. https://doi.org/10.1029/2001JE001583

Schwinger S. and Zhu, M. -H. (2018). Redistribution of titanium in the lunar mantle by giant impact-induced melting, LPSC, \#2343.

Shearer, C. K. (2006). Thermal and magmatic evolution of the Moon. Reviews in Mineralogy and Geochemistry, 60(1), 365-518. https://doi. org/10.2138/rmg.2006.60.4

Shuvalov, V. (2011). Ejecta deposition after oblique impacts: An influence of impact scale. Meteoritics and Planetary Science, 46(11), 1713-1718. https://doi.org/10.1111/j.1945-5100.2011.01259.x

Smith, D. E., Zuber, M. T., Jackson, G. B., Cavanaugh, J. F., Neumann, G. A., Riris, H., et al. (2010). The Lunar Orbiter Laser Altimeter investigation on the Lunar Reconnaissance Orbiter Mission. Space Science Reviews, 150(1-4), 209-241. https://doi.org/10.1007/s11214009-9512-y

Spohn, T., et al. (2001). The longevity of lunar volcanism: implications of thermal evolution calculations with 2D and 3D mantle convection models. Icarus, 149(1), 54-65. https://doi.org/10.1006/icar.2000.6514

Spudis, P. D. (1993). The geology of multi-ring basins, (p. 264). Cambridge, U. K: Cambridge Univ. Press.

Stöffler, D. (1972). Deformation and transformation of rock-forming minerals by natural and experimental shock processes, I. Behavior of minerals under shock compression. Fortschritte der Mineralogie, 49, 50-133.

Strom, R. G., Malhotra, R., Ito, T., Yoshida, F., \& Kring, D. A. (2005). The origin of planetary impactors in the inner solar system. Science, 309(5742), 1847-1850. https://doi.org/10.1126/science.1113544

Thompson, S. L. \& Lauson, H. S. (1972). Improvements in the CHART D radiation-Hydrodynamic Code III: Revised Analytic Equations of State. Sandia National Laboratory Report, SC-RR-71 0714. pp113.

Toksöz, M. N., Dainty, A. M., Solomon, S. C., \& Anderson, K. R. (1974). Structure of the Moon. Reviews of Geophysics, $12(4), 539-567$. https://doi.org/10.1029/RG012i004p00539

Touboul, M., Puchtel, I. S., \& Walker, R. J. (2015). Tungsten isotopic evidence for disproportional late accretion to the Earth and Moon. Nature, 520(7548), 530-533. https://doi.org/10.1038/nature14355

Trinquier, A., Elliott, T., Ulfbeck, D., Coath, C., Krot, A. N., \& Bizzarro, M. (2009). Origin of nucleosynthetic isotope heterogeneity in the solar protoplantary disk. Science, 324(5925), 374-376. https://doi.org/10.1126/science.1168221

Vaughan, W. M., \& Head, J. W. (2014). Impact melt differentiation in the South Pole-Aitken basin: Some observations and speculations. Planetary and Space Science, 91, 101-106. https://doi.org/10.1016/j.pss.2013.11.010

Vaughan, W. M., Head, J. W., Wilson, L., \& Hess, P. C. (2013). Geology and petrology of enormous volumes of impact melt on the Moon: A case study of the Orientale basin impact melt sea. Icarus, 223(2), 749-765. https://doi.org/10.1016/j.icarus.2013.01.017

Walker, R. J. (2009). Highly siderophile elements in the Earth, Moon and Mars: Update and implications for planetary accretion and differentiation. Chemie der Erde-Geochemistry, 69(2), 101-125. https://doi.org/10.1016/j.chemer.2008.10.001

Walker, R. J., Horan, M. F., Shearer, C. K., \& Papike, J. J. (2004). Low abundances of highly Siderophile elements in the lunar mantle: Evidence for prolonged late accretion. Earth and Planetary Science Letters, 224(3-4), 399-413. https://doi.org/10.1016/j. epsl.2004.05.036

Warren, P. H. (1985). The magma ocean concept and lunar evolution. Annual Review of Earth and Planetary Sciences, 13(1), 201-240. https://doi.org/10.1146/annurev.ea.13.050185.001221

Warren, P. H., Claeys, P., \& Cedillo-Pardo, E. (1996). In G. Ryder, D. Fastovsky, \& S. Gartner (Eds.), The Cretaceous-Tertiary event and other catastrophes in Earth history. Boulder, Colorado: (Geological Society of America, Special Paper 307).

Wasson, J. T., \& Warren, P. H. (1980). Contribution of the mantle to lunar asymmetry. Icarus, 44(3), 752-771. https://doi.org/10.1016/00191035(80)90142-6

Whitaker, E. A. (1981). The lunar Procellarum basin. Proceedings of Lunar and Planetary Science, 12A, $105-111$.

Wiechert, U., et al. (2001). Oxygen isotopes and the Moon-forming giant impact. Science, 249, 345-348.

Wieczorek, M. A. (2006). The constitution and structure of the lunar interior. Reviews in Mineralogy and Geochemistry, 60(1), 221-364. https://doi.org/10.2138/rmg.2006.60.3

Wieczorek, M. A., Neumann, G. A., Nimmo, F., Kiefer, W. S., Taylor, G. J., Melosh, H. J., et al. (2013). The crust of the Moon as seen by GRAIL. Science, 339(6120), 671-675. https://doi.org/10.1126/science.1231530

Wieczorek, M. A., \& Phillips, R. J. (2000). The "Procellarum KREEP Terrane": Implications for mare volcanism and lunar evolution. Journal of Geophysical Research, 105(E8), 20,417-20,430. https://doi.org/10.1029/1999JE001092

Wilhelms, D. E. (1987). The Geologic History of the Moon. USGS Professional Paper 1348, Washington.

Wilhelms, D. E. \& McCauley, J. F. (1971). Geologic map of the nearside of the Moon. I-703, USGS, Washington D. C.

Wood, J. A. (1973). Bombardment as a cause of the lunar asymmetry. Moon, 8(1-2), 73-103. https://doi.org/10.1007/BF00562751 
Wünnemann, K., Collins, G. S., \& Melosh, H. J. (2006). A strain-based porosity model for use in hydrocode simulations of impacts and implications for transient-crater growth in porous targets. Icarus, 180(2), 514-527. https://doi.org/10.1016/j.icarus.2005.10.013

Wünnemann, K., Zhu, M. -. H., \& Stöffler, D. (2016). Impacts into quartz sand: crater formation, shock metamorphism, and ejecta distribution in laboratory experiments and numerical models. Meteoritics \& Planetary Science, 51, 1762-1794.

Yamamoto, S., Nakamura, R., Matsunaga, T., Ogawa, Y., Ishihara, Y., Morota, T., et al. (2010). Possible mantle origin of olivine around lunar impact basins detected by SELENE. Nature Geoscience, 3(8), 533-536. https://doi.org/10.1038/ngeo897

Yamamoto, S., Nakamura, R., Matsunaga, T., Ogawa, Y., Ishihara, Y., Morota, T., et al. (2012). Massive layer of pure anorthosite on the Moon. Geophysical Research Letters, 39, L13201. https://doi.org/10.1029/2012GL052098

Yamamoto, S., Nakamura, R., Matsunaga, T., Ogawa, Y., Ishihara, Y., Morota, T., et al. (2015). Featureless spectra on the Moon as evidence of residual lunar primordial crust. Journal of Geophysical Research: Planets, 120, 2190-2205. https://doi.org/10.1002/ $2015 \mathrm{je} 004935$

Young, E. D., Kohl, I. E., Warren, P. H., Rubie, D. C., Jacobson, S. A., \& Morbidelli, A. (2016). Oxygen isotopic evidence for vigorous mixing during the Moon-forming giant impact. Science, 351(6272), 493-496. https://doi.org/10.1126/science.aad0525

Zhang, J. J., Dauphas, N., Davis, A. M., Leya, I., \& Fedkin, A. (2012). The proto-Earth as a significant source of lunar material. Nature Geoscience, 5(4), 251-255. https://doi.org/10.1038/ngeo1429

Zhang, N., et al. (2013). A 3-D numerical study of the thermal evolution of the Moon after cumulate mantle overturn: The importance of rheology and core solidification. Journal of Geophysical Research: Planets, 118, 1789-1804. https://doi.org/10.1002/ jgre. 20121

Zhong, S. J., et al. (2000). A dynamic origin for the global asymmetry of lunar mare basalt. Earth and Planetary Science Letters, 177(3-4), 131-140. https://doi.org/10.1016/S0012-821X(00)00041-8

Zhu, M. -. H., et al. (2013). Potassium map from Chang'E-2 constraints the impact of Crisium and Orientale basin on the Moon. Scientific Reports, 3(1), 1611. https://doi.org/10.1038/srep01611

Zhu, M. -H., et al. (2015). Numerical modeling of the ejecta distribution and formation of the Orientale basin. Journal of Geophysical Research: Planets, 120, 2118-2134. https://doi.org/10.1002/2015je004827

Zhu, M. -H., et al. (2017). Effects of Moon's thermal state on the impact basin ejecta distribution. Geophysical Research Letters, 44, 11,292-11,300. https://doi.org/10.1002/2017gl075405

Zuber, M. T., Smith, D. E., Lemoine, F. G., \& Neumann, G. A. (1994). The shape and internal structure of the Moon from the Clementine mission. Science, 266(5192), 1839-1843. https://doi.org/10.1126/science.266.5192.1839

Zuber, M. T., Smith, D. E., Watkins, M. M., Asmar, S. W., Konopliv, A. S., Lemoine, F. G., et al. (2013). Gravity field of the Moon from the Gravity Recovery and Interior Laboratory (GRAIL) mission. Science, 339(6120), 668-671. https://doi.org/10.1126/science.1231507 